Alma Mater Studiorum - Università di Bologna DEPARTMENT OF ECONOMICS

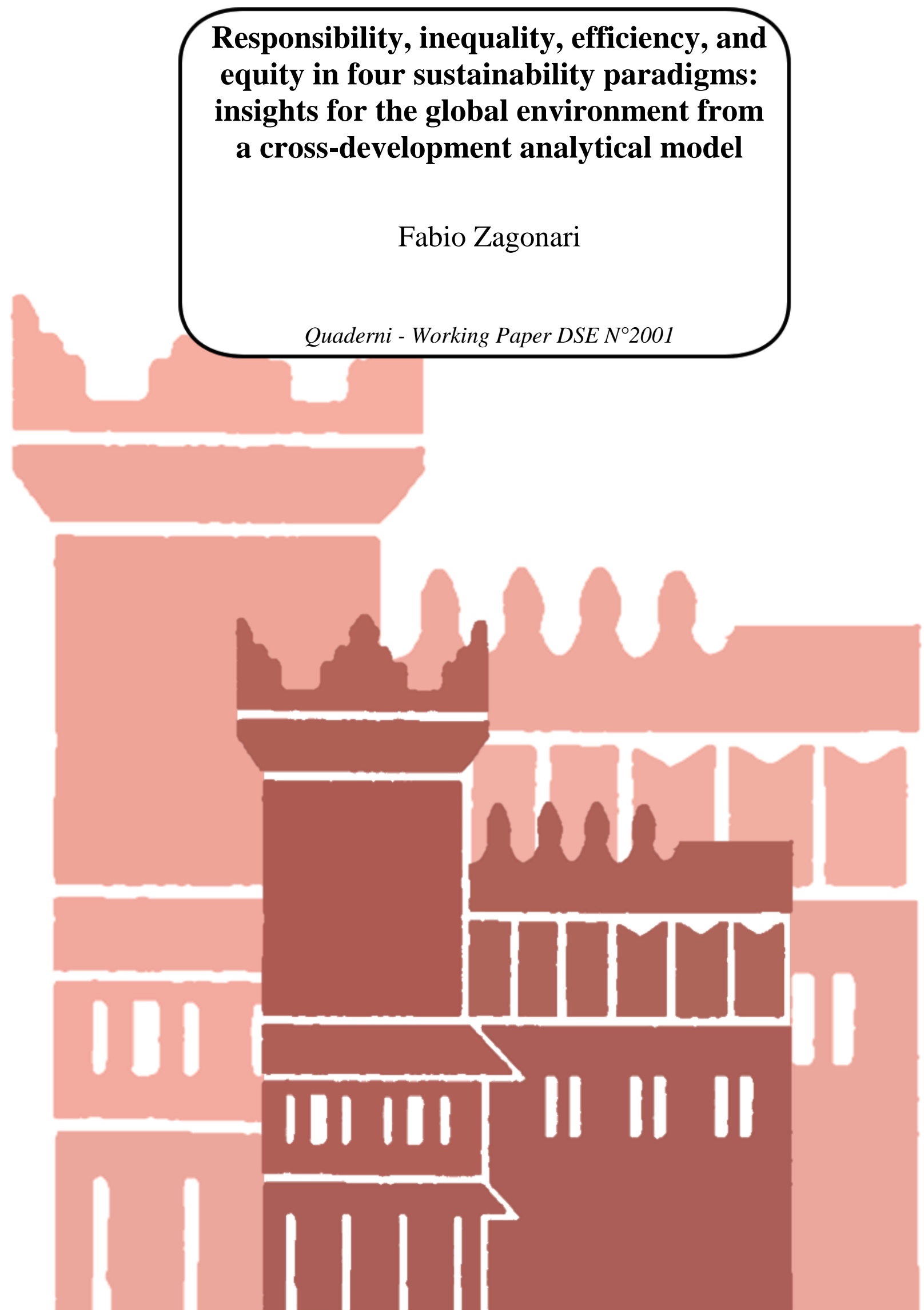




\title{
Responsibility, inequality, efficiency, and equity in four sustainability paradigms: insights for the global environment from a cross-development analytical model
}

\author{
Fabio Zagonari \\ Dipartimento di Scienze Economiche, Università di Bologna, via Angherà 22, 47900 Rimini (Italy) \\ Phone: 00390541434135 Fax: 00390541434120 E-mail: fabio.zagonari@unibo.it
}

\begin{abstract}
This paper develops a theoretical framework to assess the feasibility of global environmental sustainability solutions based on one or more value changes. The framework represents four sustainability paradigms (weak sustainability WS, a-growth AG, de-growth DG, strong sustainability SS) and five value changes (i.e., a sense of responsibility for nature $\beta$, future generations $\gamma$, or current generations in developing countries $\delta$; aversion to inequality for current generations $\varepsilon$ or future generations $\zeta$ ). It defines solutions in terms of consumption, environment use, and welfare for representative individuals in both developed (OECD) and developing (nonOECD) countries. Solutions are characterised by efficiency (i.e., Pareto and Kaldor-Hicks) with respect to welfare and by intra- and inter-generational equality for consumption, environment use, and welfare, by confirming internal consistency and consistency with alternative equity approaches for utilitarianism (i.e., Harsanyi), egalitarianism (i.e., Arneson for welfare; Dworkin for consumption or environment use; Sen for consumption and environment use), and contractarianism (i.e., Rawls). Theoretical and operational insights are described for alternative sustainability paradigms and equity approaches. In terms of feasibility, by considering improved technology $\theta$, decreased population $\eta$, and modified consumption $\alpha$, the ordering is $\gamma>\delta>\varepsilon>\zeta$ and AG $>$ SS $>$ DG > WS: $\beta$ is unfeasible. In terms of internal consistency, $\gamma>\delta=\varepsilon=\zeta$ and SS $>$ AG $>$ DG: WS is internally inconsistent. In terms of consistency with an equity approach, $\gamma>\delta=\zeta>\varepsilon$ and SS AG $>\mathrm{DG}>\mathrm{WS}$.
\end{abstract}

\section{Keywords}

Weak sustainability; a-growth; de-growth; strong sustainability; duty; inequality; efficiency; equity

JEL classification 


\section{Introduction}

Four main sustainability paradigms have been suggested in the literature (Zagonari, 2016): weak sustainability, a-growth, de-growth, and strong sustainability. Note that in this context the economic general equilibrium framework is similar to weak sustainability, whereas the ecosystem services framework is close to strong sustainability.

Two main value changes have been evoked to achieve sustainability: a sense of responsibility, whether this is for nature (Pedersen, 2015; Saniotis, 2012; Van der Werff et al., 2013) or for current and future generations (Caselles, 2013; Koukouzelis, 2012); and an aversion to inequality, whether this is with respect to current or future generations (Golub et al., 2013; Kopnina, 2016). Note that improved environmental technology, a decreased world population, and modified consumption patterns can be considered here as context changes for any combination of paradigms and values.

The purpose of this paper is to develop a model for the four sustainability paradigms within a single framework that accounts for changes in the five values (a sense of responsibility for nature, for current generations, or for future generations; an aversion to intra- or inter-generational inequality). The goal is to assess the feasibility of global environmental sustainability solutions that depend on changes in one or more of these values. In particular, analytical and numerical solutions for cases based on extreme and estimated parameter values will be characterised using data on the consumption level, the direct and indirect use of Earth's environmental resources (hereafter, environment use), and the welfare level for representative individuals in both developed countries (i.e., the 35 OECD countries) and developing countries (i.e., the non-OECD countries). This will rank solutions in terms of feasibility classes: a reduction in welfare by $>25 \%$, by 12.5 to $25 \%$, and by $<12.5 \%$ will be considered unfeasible, slightly feasible, and moderately feasible, respectively, whereas an increase in welfare will be considered feasible.

Moreover, these characterisations will let us identify efficient solutions (i.e., Pareto and KaldorHicks efficiency with respect to welfare) and measure equality (i.e., inequalities with respect to consumption, environment use, and welfare) at both intra- and inter-generational levels. This will reveal the internal consistency of the solutions with the assumptions of the four sustainability paradigms with respect to equality (e.g., weak sustainability cannot be linked to a large aversion to inequality) and efficiency (e.g., weak sustainability must be coupled with Kaldor-Hicks efficiency). Finally, these calculations will identify which sustainability solution is consistent with a utilitarian approach (i.e., Harsanyi), an egalitarian approach (i.e., Arneson for welfare; Dworkin for consumption or environment use; Sen for consumption and environment use), or a contractarian approach (i.e., Rawls) (Habib, 2013). This will characterize the sustainability solutions in terms of distributive justice (hereafter, equity).

In other words, this study can be read from a normative perspective so that, for a given sustainability paradigm or approach to equity, the framework defines which global environmental sustainability conditions should be achieved, if any. Alternatively, this study can be read from a positive perspective so that, for any sustainability paradigm or approach to equity, it identifies which value changes (i.e., demand policies vs. production policies such as taxes and standards) are crucial to meet sustainability conditions.

Note that all insights about feasible sustainability for the current generation are based on per capita data for representative individuals in OECD and non-OECD countries, weighted according to the country's proportion of the world's population. Moreover, sustainability conditions are checked for the main context changes (i.e., improved technology, decreased population, and modified consumption). Finally, a current, globally representative individual is compared with a future globally representative individual in terms of inter-generational equity and efficiency, without splitting future generations into OECD and non-OECD countries. 


\section{Paradigms, concepts, and approaches}

This section concisely defines the four sustainability paradigms, efficiency concepts, and equity approaches identified in the Introduction.

A sustainability solution is Pareto-efficient if current generations in both OECD and non-OECD countries obtain greater welfare than in the status quo situation. In other words, there are no losers. A sustainability solution is Kaldor-Hicks efficient if current OECD and non-OECD generations together obtain greater welfare than in the status quo situation so that the losers can potentially receive compensation from the winners. A sustainability solution reduces inequalities between the current OECD and non-OECD generations in terms of consumption, environment use, or welfare level if the Gini index for one of these variables is smaller than the Gini index for the same variable in the status quo situation; this situation is defined as Gini-equitable. A sustainability solution improves the conditions for the current non-OECD generation in terms of consumption, environment use, or welfare if the minimum value of one variable is larger than its value in the status quo situation; this situation is defined MaxMin-equitable.

The main assumptions behind weak sustainability (i.e., development that meets the needs of the present generation without compromising the ability of future generations to meet their own needs) can be summarized as follows (Schlör et al., 2015): needs are used as the unit of measurement; the same weights are used for current and future generations; and there is unconditional substitution among current economic, social, and environmental forms of capital at both intra- and intergenerational levels. A sustainability solution is consistent with the weak sustainability paradigm if it is at least Kaldor-Hicks efficient, and if it assumes small aversion to inter- and intra-generational inequality.

A-growth is an ecological and economic strategy focused on indifference to or neutrality about the economic level and growth, with both the economic level and growth considered to be non-robust and unreliable indicators of social welfare and progress (Van den Bergh, 2010, 2011). It can be characterised as follows: welfare is used as the unit of measurement, as deduced from the aim of moving from wrong prices that result from the many neglected non-market transactions (e.g., informal activities and relationships) and the many unpriced environmental effects to the right prices (i.e., prices that account for both non-market and unpriced values); different weights are used for current OECD and non-OECD generations; and substitution between forms of capital is possible. A sustainability solution is consistent with the a-growth paradigm if it is Gini-equitable in welfare, and if it assumes small aversion to inter-generational and intra-generational inequality.

De-growth is an ecological and economic perspective based on achieving a socially sustainable and equitable reduction (and eventually stabilization) of the materials and energy that a society extracts, processes, transports, distributes, consumes, and returns to the environment as wastes (Kallis, 2011; Kallis et al., 2012). It can be characterised as follows: happiness is the unit of measurement, with a priority on meeting the needs of the poorest individuals, as deduced from the aim of introducing a basic income; the same weight is assigned to current and future generations; and substitution among forms of capital is acceptable. A sustainability solution is consistent with the de-growth paradigm if it is MaxMin-equitable in welfare and if it assumes a large aversion to inter- and intra-generational inequality.

The main assumptions behind strong sustainability (i.e., a development that allows future generations to access the same amount of natural resources and the same environmental status as the current generation) can be summarized as follows (Jain \& Jain, 2013): requirements for some incommensurable categories as unit of measurement; possibly assignment of different weights to current and future generations; and no substitution between current or future forms of capital, with natural and physical or social capital considered to be complementary. A sustainability solution is consistent with the strong sustainability paradigm if it is Gini-equitable for consumption and environment use, and if it assumes a large aversion to inter- and intra-generational inequality.

Utilitarianism, in the version considered here (Harsanyi, 1982) can be characterised as follows: equally weighting everyone's welfare, with welfare defined as the satisfaction of rational, well- 
informed, and self-interested preferences, by maximising the total social welfare. A sustainability solution is consistent with the utilitarian approach if it is Pareto-efficient or Kaldor-Hicks efficient and if it assumes a small aversion to inter- and intra-generational inequality.

Egalitarianism, in the main three alternative versions that I focus on here, can be summarised as follows: it involves (1) levelling of resources or primary goods, as in Dworkin (1981); (2) equalising capabilities, as in Sen (1993); or (3) equalising opportunities for welfare, as in Arneson (1989). A sustainability solution is consistent with these egalitarian approaches if it assumes a large aversion to inter- and intra-generational inequality, and if it is Gini-equitable in consumption or environment use for case 1, Gini-equitable in consumption and environment use for case 2, and Gini-equitable in welfare for case 3.

Contractarianism, in the version considered here (Rawls, 1971), can be characterised as follows: it arranges social and economic inequalities to the greatest benefit of the least advantaged people by opening offices and positions to everybody. A sustainability solution is consistent with the contractarian approach if it is MaxMin-equitable for consumption, environment use, or welfare, and if it assumes a large aversion to inter- and intra-generational inequality.

Note that my analysis disregards libertarian approaches, both in terms of positive rights (Lomasky, 1987) and negative rights (Nozick, 1974), because it is arguable whether future generations or nature have rights in this context (Gosseries, 2008). Moreover, sustainability solutions in terms of value changes could be turned into policy suggestions; for example, a sense of responsibility for nature, for future generations, or for current generations could be translated into expenditures for environmental protection, environmental $\mathrm{R} \& \mathrm{D}$, and aids for poor people. Finally, equality is assumed to be instrumental (Kershnar \& Purves, 2016), since a value is attached to consequences for people.

\section{The model}

This section provides simple formalisations for the four sustainability paradigms identified in Section 2 with the goal of requiring as little data as possible about representative individuals in OECD and non-OECD countries. For simplicity, and with full recognition that this approach ignores some exceptions, I have used the subscript $\mathrm{N}$ (northern hemisphere) to label parameters for OECD countries and the subscript $S$ (southern hemisphere) for non-OECD countries. Appendix I lists all the abbreviations used in this model.

Let us assume that $E_{\mathrm{N}}$ and $E_{\mathrm{S}}$ identify the per capita use of the global environment by the current OECD and non-OECD generations, respectively. The per capita equilibrium level consistent with the current world population is $\eta$. Let us assume that $X_{\mathrm{N}}, X_{\mathrm{S}}$, and $X_{\mathrm{F}}$ identify the per capita consumption levels in the OECD current generation, non-OECD current generation, and the future generation, respectively. Thus, the use of the environment for the OECD current generation is given by $E_{\mathrm{N}}=\theta_{\mathrm{N}} X_{\mathrm{N}}$, for the non-OECD current generation is given by $E_{\mathrm{S}}=\theta_{\mathrm{S}} X_{\mathrm{S}}$, and for the future generation is given by $E_{\mathrm{F}}=\theta_{\mathrm{F}} X_{\mathrm{F}}$, where $\theta_{\mathrm{N}}, \theta_{\mathrm{S}}$, and $\theta_{\mathrm{F}}$ represent the use of the environment for each consumption unit for the OECD current generation, non-OECD current generation, and the future generation, respectively: $\theta_{\mathrm{N}}$ and $\theta_{\mathrm{S}}$ will be set at current values based on the current technology, and then simulated as smaller than current values to analyse the impacts of technological improvement.

Two main sustainability conditions can be formalised. The weighted sustainability condition requires that the use of the environment must be weighted according to the proportions of the global population in the OECD and non-OECD countries ( $p_{\mathrm{N}}$ and $p_{\mathrm{S}}$, respectively):

$$
E_{\mathrm{C}}=p_{\mathrm{N}} E_{\mathrm{N}}+p_{\mathrm{S}} E_{\mathrm{S}}
$$

Where $E_{\mathrm{C}}$ stands for the total weighted use of the environment by the current generation. The nonweighted sustainability condition requires that the use of the environment must be averaged between the representative individuals in the OECD and non-OECD countries:

$$
E_{\mathrm{C}}=1 / 2 E_{\mathrm{N}}+1 / 2 E_{\mathrm{S}}
$$

Thus, in terms of consumption levels, these sustainability conditions become, respectively:

$$
\eta=E_{\mathrm{C}}=p_{\mathrm{N}} \theta_{\mathrm{N}} X_{\mathrm{N}}+p_{\mathrm{S}} \theta_{\mathrm{S}} X_{\mathrm{S}}
$$


And

$$
\eta=E_{\mathrm{C}}=1 / 2 \theta_{\mathrm{N}} X_{\mathrm{N}}+1 / 2 \theta_{\mathrm{S}} X_{\mathrm{S}}
$$

I will use the non-weighted sustainability condition for the strong sustainability paradigm, and the weighted sustainability condition for the other sustainability paradigms. Let us assume that the utility for the future generation $\left(U_{\mathrm{F}}\right)$ depends only on the consumption level:

$$
U_{\mathrm{F}}=X_{\mathrm{F}}^{\alpha \mathrm{F}}
$$

Where $\alpha_{F}$ represents the future preference for consumption. Indeed, I optimistically assume that socioeconomic development will gradually raise non-OECD countries to the same level as the OECD countries so that there is no future non-OECD generation and, consequently, future OECD countries cannot be concerned about non-OECD countries. Next, I assume that the use of the global environment is in its long-run equilibrium so people do not need to feel a responsibility to preserve the environment for subsequent generations. Let us assume that the welfare of the current nonOECD generation $\left(U_{\mathrm{S}}\right)$ depends on their consumption level, the use of the environment, and the welfare of the future generation:

$$
U_{\mathrm{S}}=X_{\mathrm{S}}{ }^{\alpha \mathrm{S}} E_{\mathrm{S}}{ }^{-\beta \mathrm{S}} U_{\mathrm{F}}{ }^{\gamma \mathrm{S}}
$$

Where $\alpha_{S}$ represents the preference for consumption, $\beta_{S}$ represents the degree of concern over use of the environment, and $\gamma_{S}$ represents the degree of concern for future generations. Indeed, the current non-OECD generation is less affluent, and cannot afford to be heavily concerned with people from OECD countries. Let us assume that the welfare of the current OECD generation depends on their consumption level, their use of Earth resources, the welfare of the future generation, and the welfare of the current non-OECD generation:

$$
U_{\mathrm{N}}=X_{\mathrm{N}}^{\alpha \mathrm{N}} E_{\mathrm{N}}{ }^{-\beta \mathrm{N}} U_{\mathrm{F}}^{\gamma \mathrm{N}} U_{\mathrm{S}}^{\delta \mathrm{N}}
$$

Where $\alpha_{\mathrm{N}}$ represents the preference for consumption, $\beta_{\mathrm{N}}$ represents concern over the use of Earth resources, $\gamma_{\mathrm{N}}$ represents concern for future generations, and $\delta_{\mathrm{N}}$ represents the concern for the current non-OECD generation (Lauwers, 2012). In other words, both OECD and non-OECD countries are assumed to be concerned about their own environment (i.e., $E_{\mathrm{N}}$ and $E_{\mathrm{S}}$, respectively) rather than about the overall world environment (i.e., $E_{\mathrm{C}}=p_{\mathrm{N}} E_{\mathrm{N}}+p_{\mathrm{S}} E_{\mathrm{S}}$ ). Indeed, non-OECD countries might not be concerned about the environment in the OECD countries, whereas OECD countries are assumed to be concerned about consequences of the use of the environment on the welfare level in non-OECD countries. Moreover, the concern for the total environment seems to be more plausible in the case of a specified common environment (e.g., a closed sea such as the Baltic Sea) to be shared by a specified group of countries. Appendix II presents a formalisation of this analysis in the case of a shared common environment. Finally, maximising the total welfare first and then accounting for sustainability conditions (rather than in the opposite sequence) would lead to higher production and consumption in the more efficient OECD countries, at levels even larger than current production and consumption, with sustainability conditions for the weak sustainability and a-growth paradigms being less feasible.

Note that a logarithmic transformation of the Cobb-Douglas utility functions permits analytical solutions. Moreover, apart from nature, welfare could be directly affected by other types of capital such as social, physical, and human capital, where these forms of capital, like nature, contribute to achieving a given consumption level. Finally, each parameter attached to an item of the CobbDouglas utility function (e.g., $\alpha$ to consumption, $\beta$ to the environment, $\gamma$ to the welfare of future generations, $\delta$ to welfare of non-OECD current generations) can be related to the proportion of the budget spent to purchase it. Let us assume that a representative individual in the current generation is concerned about welfare inequality between OECD and non-OECD countries:

$$
U_{\mathrm{C}}=\left[\left(p_{\mathrm{N}} U_{\mathrm{N}}\right)^{1-\varepsilon}+\left(p_{\mathrm{S}} U_{\mathrm{S}}\right)^{1-\varepsilon}\right]^{1 /(1-\varepsilon)}
$$

Where $\varepsilon$ is the degree of aversion to intra-generational inequality (Asheim et al., 2012). Thus, the overall utility is given by:

$$
U=\left[U_{\mathrm{C}}{ }^{1-\zeta}+U_{\mathrm{F}}{ }^{1-\zeta}\right]^{1 /(1-\zeta)}
$$


Where $\zeta$ is the degree of aversion to inter-generational inequality. Alternatively, a representative individual in the current generation could be concerned about inequality in use of the global environment between OECD and non-OECD countries:

Thus, the overall welfare would be given by:

$$
W_{\mathrm{C}}=\left[E_{\mathrm{N}}^{1-\varepsilon}+E_{\mathrm{S}}^{1-\varepsilon}\right]^{1 /(1-\varepsilon)}
$$

$$
W=\left[E_{\mathrm{C}}{ }^{1-\zeta}+E_{\mathrm{F}}{ }^{1-\zeta}\right]^{1 /(1-\zeta)}
$$

Note that the time discount rate is assumed to be 0 , as this is the only value that is consistent with long-run equilibria. Moreover, each social utility or welfare function can be linked to an Atkinson inequality index, in which parameters $\varepsilon$ and $\zeta$ have the same meaning. Finally, extreme values of $\varepsilon$ and $\zeta$ (i.e., at 0 and 1 ) permit analytical solutions.

Many theoretical definitions of the four sustainability paradigms can be suggested (Aznar-Marquez $\&$ Ruiz-Tamarit, 2016). Here, I will apply the analytical definitions summarised in Table 1. In other words, OECD and non-OECD countries are both assumed to adopt a cooperative rather than a noncooperative attitude, leading (for example) to a Nash equilibrium. This is realistic because currently, there is no coalition of OECD countries playing against the interests of non-OECD countries. Moreover, a non-cooperative context seems to be more plausible in the case of a specified group of countries exploiting a common environment. See Appendix II for formalisation of this analysis in the case of a shared common environment. Finally, referring to a non-cooperative context disregards the aversion to inter-generational inequality.

Table 1. The analytical definitions of the four sustainability paradigms. $U$, overall utility in terms of consumption; $U_{\mathrm{F}}$, utility for the future generation; $U_{\mathrm{C}}$, total weighted utility for the current generation; $E_{\mathrm{c}}$, total weighted use of the environment by the current generation; $E_{\mathrm{F}}$, use of the environment by the future generation; $X_{\mathrm{C}}$, total weighted consumption by the current generation; $X_{\mathrm{F}}$, per capita consumption by the future generation; $W$, overall welfare in terms of the environment.

\begin{tabular}{|c|c|}
\hline Paradigm & Analytical definition \\
\hline Weak sustainability & Max $U$ s.t. $U_{\mathrm{F}} \geq U_{\mathrm{C}}$ \\
\hline A-growth & $\operatorname{Max} U$ s.t. $E_{\mathrm{C}} \leq E_{\mathrm{F}}$ \\
\hline De-growth & $\operatorname{Min} X_{\mathrm{C}}$ s.t. $U_{\mathrm{F}} \geq U_{\mathrm{C}}$ \\
\hline Strong sustainability & $\operatorname{Max} W$ s.t. $E_{\mathrm{C}} \leq E_{\mathrm{F}}$ \\
\hline
\end{tabular}

Note that the analytical definition of de-growth does not depend on $E_{\mathrm{N}}$. This is consistent with the main critiques to this paradigm. Moreover, the four sustainability paradigms share couples of conditions: for example, Max $U$ is shared by weak sustainability and a-growth; $U_{\mathrm{F}} \geq U_{\mathrm{C}}$ is shared by weak sustainability and de-growth; and $E_{\mathrm{C}} \leq E_{\mathrm{F}}$ is shared by a-growth and strong sustainability. Finally, the analytical definition of strong sustainability assumes that the parameters $\varepsilon$ and $\zeta$ are set at 1. This is consistent with the main feature of this paradigm (i.e., maximum aversion to inequality).

By solving for the sustainability requirements for consumption level and environment use in nonOECD countries, and by using these expressions as inputs for the maximisation or minimisation problems that identify the four sustainability paradigms, it becomes possible to characterise these problems in terms of the variables for the OECD countries, for which solutions for $X_{\mathrm{N}}$ and $E_{\mathrm{N}}$ represent solutions for the world that meet global sustainability conditions.

Note that weak inequalities will be solved as equalities. Moreover, other changes could affect equilibria: I will therefore perform a ceteris paribus analysis. Finally, dynamic stability conditions will not be considered, and static sustainability equilibria will be obtained.

\section{Data and normalisations}

Some parameters of the model developed in section 3 can be directly estimated. In particular, the proportions of the world's current population in OECD and non-OECD countries, based on World Bank world development indicators (http://data.worldbank.org) data for 2012, are $p_{\mathrm{N}}=0.18$ and $p_{\mathrm{S}}$ $=0.82$. If the per capita use of the global environment is measured by the ecological footprint (i.e., 
the biologically productive area needed to provide everything an individual uses), sustainability of a representative individual for the world at the current population level requires $E_{\mathrm{F}}$ to be at $\eta=1.7$ ha (http://www.footprintnetwork.org), whereas the values for use of the environment in OECD and non-OECD countries, based on data for 2012, are 5.74 and 2.15 ha, respectively. The actual individual consumption as a percentage of GDP is available for each OECD country, with an average at $71.1 \%$. Unfortunately, comparable data are not available for non-OECD countries, although their average is likely to be larger. Without significant loss of generality, I will assume that the per capita consumption is measured by the per capita income (i.e. GDP in USD, based on purchasing power parity [PPP]). Indeed, postponed consumption (as saving or investment) affects the welfare of future generations, but this welfare increase contributes to the current generation's utility (i.e., both $U_{\mathrm{N}}$ and $U_{\mathrm{S}}$ depend on $U_{\mathrm{F}}$ ). Moreover, consumption of imported goods (typically, in OECD countries) increases welfare where they are consumed, but their production might increase the use of the environment and so reduce welfare where they are produced and then exported (typically, in non-OECD countries). However, this welfare decrease in non-OECD countries contributes to the utility of OECD countries (i.e., $U_{\mathrm{N}}$ depends on $U_{\mathrm{S}}$ ). Finally, net exports equal net imports at the world level. Thus, the per capita consumption levels in OECD and non-OECD countries, based on world development indicators data for 2012, are US\$36 727 GDP PPP and US\$8216 GDP PPP, respectively. The current OECD generation's aversion to inequality for the current non-OECD generation $(\varepsilon)$ and the current generation's aversion to inequality for future generations $(\zeta)$ are both in the range $[0.01,0.99]$.

Some parameters of the model developed in section 3 require additional assumptions or manipulations. In particular, the future population was normalised to 1 . In other words, I compare representative individuals for the current and future world, with a change in the future population depicted by a change in the sustainable per-capita $E_{\mathrm{F}}$. Future consumption preferences are assumed to converge towards the preferences of the current OECD generation (i.e., $\alpha_{\mathrm{F}}=\alpha_{\mathrm{N}}$ ). This assumption seems to be consistent with the observed aspiration of the current non-OECD generation, whereas a future preference for consumption at a level that equals the average of current preferences seems to be more plausible in the case of a specified group of countries at similar levels of development. See Appendix II for formalisation of this analysis in the case of a shared common environment.

The future generation achieves sustainability by relying on the environmental technology currently being applied by the OECD countries (i.e., $\theta_{\mathrm{F}}=\theta_{\mathrm{N}}$ ). In other words, complete technology transfer between developed and developing nations is optimistically assumed to be implemented in the future. Indeed, some technological convergence is likely to occur, although it is impossible to quantify the degree of this convergence. Moreover, a future technology that represents an average of the capabilities of current technologies seems to be more plausible in the case of a specified group of countries at similar levels of development. See Appendix II for formalisation of this analysis in the case of a shared common environment. Finally, this assumption does not affect the solutions for the a-growth and strong sustainability paradigms, but makes the solutions for the weak sustainability and de-growth paradigms less feasible.

The remaining parameters of the model developed in section 3 can be indirectly estimated. In particular, since the benchmark scenario is characterised by OECD countries attaching the same importance (i.e., the same budget share) to consumption level, environmental preservation, the welfare of future generations, and the welfare of people in non-OECD countries; in this analysis, the budget share for consumption represents the parameter with the maximum budget share (i.e., the preference for consumption is realistically assumed not to be smaller than the concerns for the other issues), with the budget shares for all parameters summing up to 1 . On this basis, I have fixed $\alpha_{N}$ at 0.25 , and have normalised all other parameters with respect to this value (i.e., the observed budget shares are multiplied by 0.25$)$. The OECD concern about the use of the environment $\left(\beta_{\mathrm{N}}\right)$ is based on the assumption that $6.8 \%$ of GDP is devoted to the environment in OECD countries. This value was calculated by multiplying the observed average government expenditure on environmental protection as a percentage of GDP in OECD countries (i.e., 1.7\% in the OECD data; 
http://www.oecd-ilibrary.org) by 4 , to account for both public and private expenditures as well as for both direct and indirect expenditures. That is, this assumes equal expenditures for each of these four categories of expenditure. Similarly, the current OECD generation's concern for future generations $\left(\gamma_{\mathrm{N}}\right)$ is based on the average value of $4.8 \%$ of GDP devoted to green R\&D and patents in OECD countries. This percentage was calculated by multiplying the average observed expenditure on environmental $R \& D$ and patents as a percentage of GDP observed in OECD countries (i.e., $2.4 \%$ in OECD data; http://www.oecd-ilibrary.org) by 2 , to take into account both direct and indirect expenditures. That is, this assumes equal expenditures for public and private expenditures. Similarly, the current OECD generation's concern for the current non-OECD generation $\left(\delta_{\mathrm{N}}\right)$ is based on the assumption that $1.2 \%$ of GDP in OECD countries is devoted to providing development assistance. This is calculated by multiplying the average observed official government expenditure on development assistance as a percentage of GDP in OECD countries (i.e., $0.3 \%$ in OECD data; http://www.oecd-ilibrary.org) by 4 to account for both public and private expenditures as well as both direct and indirect expenditures. That is, this assumes equal expenditures for each of these four categories of expenditure.

To facilitate comparisons between the numerical simulations, without significant loss of generality, I will normalise $\beta_{\mathrm{N}}=\gamma_{\mathrm{N}}=\delta_{\mathrm{N}}=0.01$ to represent an average share of GDP of $4 \%$ (i.e., $[0.01 / 0.25] \times 100 \%)$. Unfortunately, comparable data are not available for non-OECD countries. Without significant loss of generality, I will assume that $\alpha_{S}=\alpha_{N}$ (i.e., since only three parameters are inputs for $U_{\mathrm{S}}$, this implies a greater importance attached to consumption for people in nonOECD countries) and $\beta_{\mathrm{S}}=\gamma_{\mathrm{S}}=0.005$ (i.e., the current non-OECD generation's concern over use of Earth resources and concern for future generations is assumed to be half of what is estimated for the current OECD generation). Indeed, all these figures are likely to be tiny but positive.

\section{Results}

In this section, for the sustainability paradigms described using the model developed in section 3, I will provide analytical solutions for cases based on extreme parameter values, and identify numerical solutions for cases based on the data and normalisations described in section 4 . I will do this by relying on graphs that represent the relevant conditions within the whole problem domain for the OECD consumption level and use of the environment: $X_{\mathrm{N}}$ in [0, 36.727] and $E_{\mathrm{N}}$ in [0, 5.74], where 36.727 and 5.74 are the current values. These solutions are then characterised in terms of the alternative efficiency and equity approaches by referring to the following current (i.e., status quo) values: $X_{\mathrm{F}}=10.876, E_{\mathrm{F}}=1.7, U_{\mathrm{F}}=1.81, X_{\mathrm{C}}=13.348, E_{\mathrm{C}}=2.79$, and $U_{\mathrm{C}}=1.82$.

\subsection{Corner solutions}

In this section, I search for feasible solutions arising from changes in a single preference parameter to determine whether a single change can achieve sustainability. To do so, I measure feasibility in terms of acceptable welfare losses in OECD and non-OECD countries.

For a situation in which there is no concern for nature, future generations, and non-OECD countries (i.e., $\beta_{\mathrm{N}}=\gamma_{\mathrm{N}}=\delta_{\mathrm{N}}=0$ ) in OECD countries, and in which there is no concern for nature and future generations (i.e., $\beta_{S}=\gamma_{S}=0$ ) in non-OECD countries, and for which the current generation's aversion to inequality for the current non-OECD generation and the current generation's aversion to inequality for future generations are both at their minimum (i.e., $\varepsilon=\zeta=0$ ), the analytical solutions for the four sustainability paradigms are as follows:

For weak sustainability (i.e., first-order conditions for Max $U$ and $U_{\mathrm{F}} \geq U_{\mathrm{C}}$ ):

$$
X_{\mathrm{N}}=\frac{\eta \alpha_{\mathrm{N}}}{\theta_{\mathrm{N}}\left(p_{\mathrm{N}} \alpha_{\mathrm{N}}+p_{\mathrm{S}} \alpha_{\mathrm{S}}\right)} \text { and } X_{\mathrm{N}} \leq \exp ^{\frac{U \mathrm{~F}}{\alpha \mathrm{N} p \mathrm{~N}}} X_{\mathrm{S}}^{-\frac{\alpha \mathrm{S} p \mathrm{~S}}{\alpha \mathrm{N} p \mathrm{~N}}}
$$

For a-growth (i.e., first-order conditions for Max $U$ and $E_{\mathrm{C}} \leq E_{\mathrm{F}}$ ):

$$
X_{\mathrm{N}}=\frac{\eta \alpha_{\mathrm{N}}}{\theta_{\mathrm{N}}\left(p_{\mathrm{N}} \alpha_{\mathrm{N}}+p_{\mathrm{S}} \alpha_{\mathrm{S}}\right)} \text { and } p_{\mathrm{N}} \theta_{\mathrm{N}} X_{\mathrm{N}}+p_{\mathrm{S}} \theta_{\mathrm{S}} X_{\mathrm{S}} \leq \eta
$$

For de-growth (i.e., $U_{\mathrm{F}} \geq U_{\mathrm{C}}$ and $X_{\mathrm{C}} \leq X_{\mathrm{F}}$ ): 


$$
X_{\mathrm{N}} \leq \exp ^{\frac{U \mathrm{~F}}{\alpha \mathrm{N} p \mathrm{~N}}} X_{\mathrm{S}}^{-\frac{\alpha \mathrm{S} p \mathrm{~S}}{\alpha \mathrm{N} p \mathrm{~N}}} \text { and } p_{\mathrm{N}} X_{\mathrm{N}}+p_{\mathrm{S}} X_{\mathrm{S}} \leq X_{\mathrm{F}}
$$

For strong sustainability (i.e., first-order conditions for Max $W$ and $E_{\mathrm{C}} \leq E_{\mathrm{F}}$ ):

$$
X_{\mathrm{N}}=\frac{\eta}{\theta_{\mathrm{N}}} \text { and } X_{\mathrm{S}}=\frac{\eta}{\theta_{\mathrm{S}}} \text { and } p_{\mathrm{N}} \theta_{\mathrm{N}} X_{\mathrm{N}}+p_{\mathrm{S}} \theta_{\mathrm{S}} X_{\mathrm{S}} \leq \eta
$$

Figure 1 illustrates the numerical solutions for these equations based on empirical data.

Figure 1. Solutions for use of the environment $\left(E_{N}\right)$ as a function of per capita consumption $\left(X_{N}\right)$ for $O E C D$ countries based on current preferences: $\alpha_{N}=\alpha_{S}=0.25, \beta_{N}=\gamma_{N}=\delta_{N}=0.01, \beta_{S}=\gamma_{S}=0.005, \varepsilon=\zeta=0.01$. The cluster of curves represents the relationship between $E_{N}$ and $X_{N}$ for four values of $\left(U_{F}-U_{C}\right) / U_{F}$, with the value decreasing from left to right. The thick decreasing curve to the right of this cluster represents the first-order conditions for Max $U$. The increasing straight line represents the border condition for $E_{\mathrm{F}} \leq \boldsymbol{E}_{\mathrm{C}}$. The horizontal straight line represents the first-order conditions for Max $W$. The highest, middle, and lowest dots are the representative OECD, world, and non-OECD individuals, respectively.

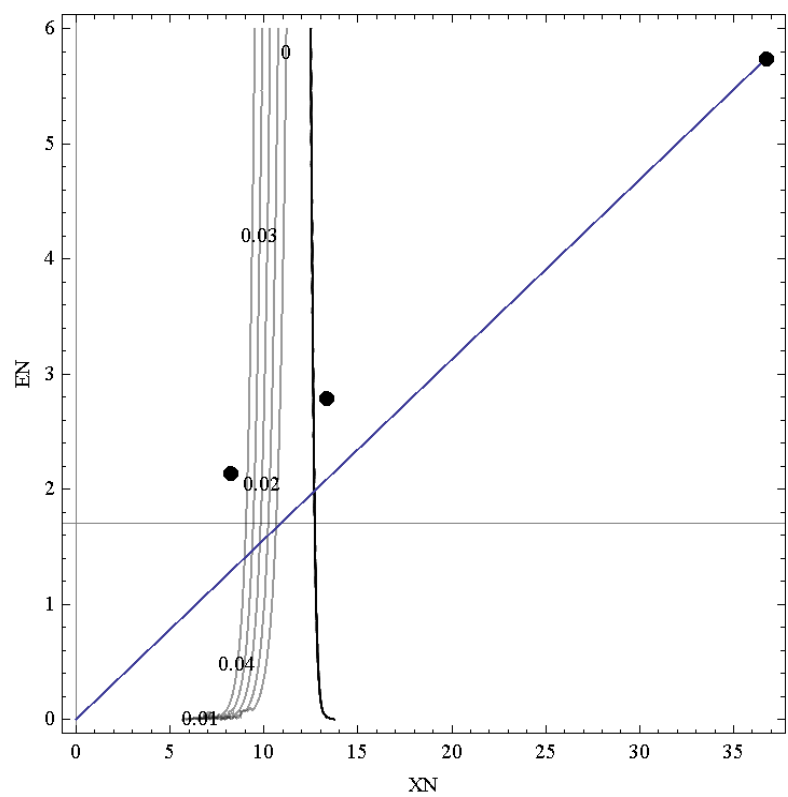

For a situation in which there is no concern for future generations and non-OECD countries (i.e., $\gamma_{\mathrm{N}}$ $=\delta_{\mathrm{N}}=0$ ) in OECD countries, and for which there is no concern for future generations (i.e., $\gamma_{S}=0$ ) in non-OECD countries, and for which the current generation's aversion to inequality for the current non-OECD generation and the current generation's aversion to inequality for future generations are both at their minimum (i.e., $\varepsilon=\zeta=0$ ), the analytical solutions for the four sustainability paradigms are as follows:

For weak sustainability (i.e., first-order conditions for Max $U$ and $U_{\mathrm{F}} \geq U_{\mathrm{C}}$ ):

$$
\begin{aligned}
& X_{\mathrm{N}}=\frac{\eta\left(\alpha_{\mathrm{N}}-\beta_{\mathrm{N}}\right)}{\theta_{\mathrm{N}}\left[p_{\mathrm{N}}\left(\alpha_{\mathrm{N}}-\beta_{\mathrm{N}}\right)+p_{\mathrm{S}}\left(\alpha_{\mathrm{S}}-\beta_{\mathrm{S}}\right)\right]} \text { and } X_{\mathrm{N}} \\
& \quad \leq \exp \frac{U \mathrm{~F}}{\overline{(\alpha \mathrm{N}-\beta \mathrm{N}) p \mathrm{~N}}}\left(p_{\mathrm{N}} \theta_{\mathrm{N}}\right)^{\frac{\beta \mathrm{N} p \mathrm{~N}}{(\alpha \mathrm{N}-\beta \mathrm{N}) p \mathrm{~N}}}\left(p_{\mathrm{S}} \theta_{\mathrm{S}}\right)^{\frac{\beta \mathrm{S} p \mathrm{~S}}{(\alpha \mathrm{N}-\beta \mathrm{N}) p \mathrm{~N}}} X_{\mathrm{S}}{ }^{-\frac{(\alpha \mathrm{S}-\beta \mathrm{S}) p S}{(\alpha \mathrm{N}-\beta \mathrm{N}) p \mathrm{~N}}}
\end{aligned}
$$

For a-growth (i.e., first-order conditions for Max $U$ and $E_{\mathrm{C}} \leq E_{\mathrm{F}}$ ):

$$
X_{\mathrm{N}}=\frac{\eta\left(\alpha_{\mathrm{N}}-\beta_{\mathrm{N}}\right)}{\theta_{\mathrm{N}}\left[p_{\mathrm{N}}\left(\alpha_{\mathrm{N}}-\beta_{\mathrm{N}}\right)+p_{\mathrm{S}}\left(\alpha_{\mathrm{S}}-\beta_{\mathrm{S}}\right)\right]} \text { and } p_{\mathrm{N}} \theta_{\mathrm{N}} X_{\mathrm{N}}+p_{\mathrm{S}} \theta_{\mathrm{S}} X_{\mathrm{S}} \leq \eta
$$

For de-growth (i.e., $U_{\mathrm{F}} \geq U_{C}$ and $X_{\mathrm{C}} \leq X_{\mathrm{F}}$ ):

$$
X_{\mathrm{N}} \leq \exp \frac{U \mathrm{~F}}{\overline{(\alpha \mathrm{N}-\beta \mathrm{N}) p \mathrm{~N}}}\left(p_{\mathrm{N}} \theta_{\mathrm{N}}\right)^{\frac{\beta \mathrm{N} p \mathrm{~N}}{(\alpha \mathrm{N}-\beta \mathrm{N}) p \mathrm{~N}}}\left(p_{\mathrm{S}} \theta_{\mathrm{S}}\right)^{\frac{\beta \mathrm{S} p \mathrm{~S}}{(\alpha \mathrm{N}-\beta \mathrm{N}) p \mathrm{~N}}} X_{\mathrm{S}}{ }^{-\frac{(\alpha \mathrm{S}-\beta \mathrm{S}) p S}{(\alpha \mathrm{N}-\beta \mathrm{N}) p \mathrm{~N}}} \text { and } p_{\mathrm{N}} X_{N}+p_{S} X_{S} \leq X_{\mathrm{F}}
$$

For strong sustainability (i.e., first-order conditions for $\operatorname{Max} W$ and $E_{\mathrm{C}} \leq E_{\mathrm{F}}$ ):

$$
X_{\mathrm{N}}=\frac{\eta}{\theta_{\mathrm{N}}} \text { and } X_{\mathrm{S}}=\frac{\eta}{\theta_{\mathrm{S}}} \text { and } p_{\mathrm{N}} \theta_{\mathrm{N}} X_{\mathrm{N}}+p_{\mathrm{S}} \theta_{\mathrm{S}} X_{\mathrm{S}} \leq \eta
$$

Figure 2 illustrates the numerical solutions based on empirical data. 
Figure 2. Solutions for use of the environment $\left(E_{\mathrm{N}}\right)$ as a function of per capita consumption $\left(X_{\mathrm{N}}\right)$ for $\mathrm{OECD}$ countries based on the perceived responsibility for nature: $\alpha_{N}=\alpha_{S}=0.25, \beta_{N}=\beta_{S}=0.73, \gamma_{N}=\delta_{N}=0.01, \gamma_{S}=0.005$, $\varepsilon=\zeta=0.01$. The cluster of curves represents the relationship between $E_{N}$ and $X_{\mathrm{N}}$ for four values of $\left(U_{\mathrm{F}}-U_{\mathrm{C}}\right) / U_{\mathrm{F}}$, with the value decreasing from top to bottom. The thick decreasing curve represents the first-order conditions for Max $U$. The increasing straight line represents the border condition for $E_{\mathrm{F}} \leq E_{\mathrm{C}}$. The horizontal straight line represents the first-order conditions for $\operatorname{Max} W$. The highest, middle, and lowest dots are the representative OECD, world, and non-OECD individuals, respectively.

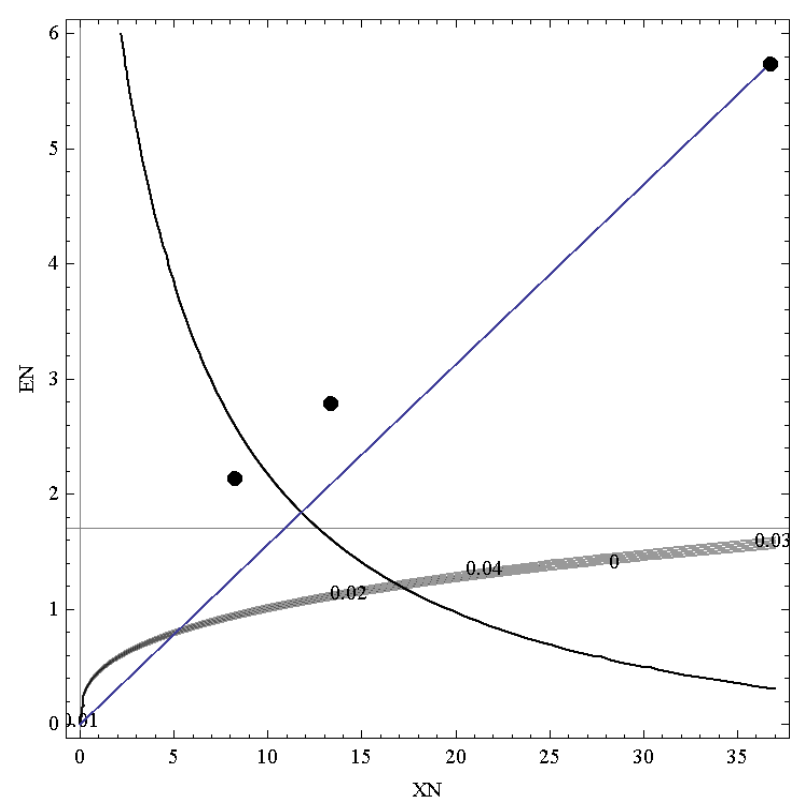

For a situation in which there is no concern for nature and non-OECD countries (i.e., $\beta_{N}=\delta_{N}=0$ ) in OECD countries, and in which there is no concern for nature (i.e., $\gamma_{S}=0$ ) in non-OECD countries, and in which the current generation's aversion to inequality for the current non-OECD generation and the current generation's aversion to inequality for future generations are both at their minimum (i.e., $\varepsilon=\zeta=0$ ), the analytical solutions for the four sustainability paradigms are as follows:

For weak sustainability (i.e., first-order conditions for Max $U$ and $U_{\mathrm{F}} \geq U_{\mathrm{C}}$ ):

$$
X_{\mathrm{N}}=\frac{\eta \alpha_{\mathrm{N}}}{\theta_{\mathrm{N}}\left(p_{\mathrm{N}} \alpha_{\mathrm{N}}+p_{\mathrm{S}} \alpha_{\mathrm{S}}\right)} \text { and } X_{\mathrm{N}} \leq \exp ^{\frac{U \mathrm{~F}}{\alpha \mathrm{N} p \mathrm{~N}}} X_{S}-\frac{\alpha \mathrm{S} p \mathrm{~S}}{\alpha \mathrm{N} p \mathrm{~N}}
$$

For a-growth (i.e., first-order conditions for Max $U$ and $E_{\mathrm{C}} \leq E F$ ):

$$
X_{\mathrm{N}}=\frac{\eta \alpha_{\mathrm{N}}}{\theta_{\mathrm{N}}\left(p_{\mathrm{N}} \alpha_{\mathrm{N}}+p_{\mathrm{S}} \alpha_{\mathrm{S}}\right)} \text { and } p_{\mathrm{N}} \theta_{\mathrm{N}} X_{\mathrm{N}}+p_{\mathrm{S}} \theta_{\mathrm{S}} X_{\mathrm{S}} \leq \eta
$$

For de-growth (i.e., $U_{\mathrm{F}} \geq U C$ and $X C \leq X_{\mathrm{F}}$ ):

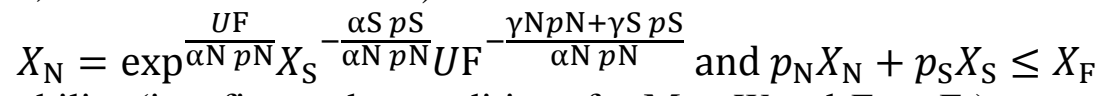

For strong sustainability (i.e., first-order conditions for $\operatorname{Max} W$ and $E_{\mathrm{C}} \leq E_{\mathrm{F}}$ ):

$$
X_{\mathrm{N}}=\frac{\eta}{\theta_{\mathrm{N}}} \text { and } X_{\mathrm{S}}=\frac{\eta}{\theta_{\mathrm{S}}} \text { and } p_{\mathrm{N}} \theta_{\mathrm{N}} X_{\mathrm{N}}+p_{\mathrm{S}} \theta_{\mathrm{S}} X_{\mathrm{S}} \leq \eta
$$

Figure 3 illustrates the numerical solutions based on empirical data. 
Figure 3. Solutions for use of the environment $\left(E_{\mathrm{N}}\right)$ as a function of per capita consumption $\left(X_{\mathrm{N}}\right)$ for OECD countries based on a concern for future generations: $\alpha_{N}=\alpha_{S}=0.25, \gamma_{N}=\gamma_{S}=0.73, \beta_{N}=\delta_{N}=0.01, \beta_{S}=0.005, \varepsilon=\zeta$ $=0.01$. The cluster of curves represents the relationship between $E_{\mathrm{N}}$ and $X_{\mathrm{N}}$ for four values of $\left(U_{\mathrm{F}}-U_{\mathrm{C}}\right) / U_{\mathrm{F}}$, with the value decreasing from left to right. The thick decreasing curve to the right of this cluster represents the first-

order conditions for Max $U$. The increasing straight line represents the border condition for $E_{\mathrm{F}} \leq E_{\mathrm{C}}$. The horizontal straight line represents the first-order conditions for Max $W$. The highest, middle, and lowest dots are the representative OECD, world, and non-OECD individuals, respectively.

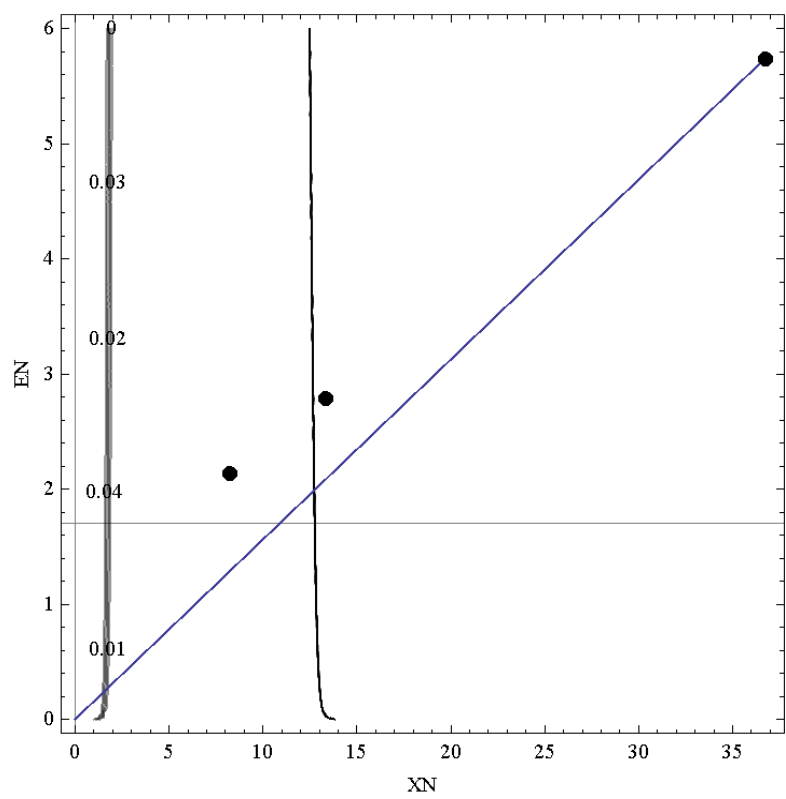

For a situation in which there is no concern for nature and future generations (i.e., $\beta_{N}=\gamma_{N}=0$ ) in OECD countries, and in which there is no concern for nature and future generations (i.e., $\beta_{\mathrm{S}}=\gamma_{\mathrm{S}}=$ 0 ) in non-OECD countries, and in which the current generation's aversion to inequality for the current non-OECD generation and the current generation's aversion to inequality for future generations are both at their minimum (i.e., $\varepsilon=\zeta=0$ ), the analytical solutions for the four sustainability paradigms are as follows:

For weak sustainability (i.e., first-order conditions for Max $U$ and $U_{\mathrm{F}} \geq U_{\mathrm{C}}$ ):

$$
X_{\mathrm{N}}=\frac{\alpha_{\mathrm{N}} \eta \ln \left[X_{\mathrm{S}}\right]}{\theta_{\mathrm{N}}\left(p_{\mathrm{N}} \delta_{\mathrm{N}}+p_{\mathrm{N}} \alpha_{\mathrm{N}} \ln \left[X_{\mathrm{S}}\right]+p_{\mathrm{S}} \alpha_{\mathrm{S}} \ln \left[X_{\mathrm{S}}\right]\right)} \text { and } X_{\mathrm{N}} \leq \alpha_{\mathrm{S}}^{-\frac{\delta \mathrm{N}}{\alpha \mathrm{N}}} \exp ^{\frac{U \mathrm{~F}}{\alpha \mathrm{N}}} X_{\mathrm{S}}^{-\frac{\alpha \mathrm{S} p \mathrm{~S}}{\alpha \mathrm{N} p \mathrm{~N}}} \ln \left[X_{\mathrm{S}}\right]^{-\frac{\delta \mathrm{N}}{\alpha \mathrm{N}}}
$$

For a-growth (i.e., first-order conditions for $\operatorname{Max} U$ and $E_{\mathrm{C}} \leq E_{\mathrm{F}}$ ):

$$
X_{\mathrm{N}}=\frac{\alpha_{\mathrm{N}} \eta \ln \left[X_{\mathrm{S}}\right]}{\theta_{\mathrm{N}}\left(p_{\mathrm{N}} \delta_{\mathrm{N}}+p_{\mathrm{N}} \alpha_{\mathrm{N}} \ln \left[X_{\mathrm{S}}\right]+p_{\mathrm{S}} \alpha_{\mathrm{S}} \ln \left[X_{\mathrm{S}}\right]\right)} \text { and } p_{\mathrm{N}} \theta_{\mathrm{N}} X_{\mathrm{N}}+p_{\mathrm{S}} \theta_{\mathrm{S}} X_{\mathrm{S}} \leq \eta
$$

For de-growth (i.e., $U_{\mathrm{F}} \geq U_{\mathrm{C}}$ and $X_{\mathrm{C}} \leq X_{\mathrm{F}}$ ):

$$
X_{\mathrm{N}}=\alpha_{\mathrm{S}}^{-\frac{\delta \mathrm{N}}{\alpha \mathrm{N}}} \exp ^{\frac{U \mathrm{~F}}{\alpha \mathrm{N} p \mathrm{~N}}} X_{\mathrm{S}}^{-\frac{\alpha \mathrm{S} p \mathrm{~S}}{\alpha \mathrm{N} p \mathrm{~N}}} \ln \left[X_{\mathrm{S}}\right]^{-\frac{\delta \mathrm{N}}{\alpha \mathrm{N}}} \text { and } p_{\mathrm{N}} X_{\mathrm{N}}+p_{\mathrm{S}} X_{\mathrm{S}} \leq X_{\mathrm{F}}
$$

For strong sustainability (i.e., first-order conditions for Max $W$ and $E_{\mathrm{C}} \leq E_{\mathrm{F}}$ ):

$$
X_{\mathrm{N}}=\frac{\eta}{\theta_{\mathrm{N}}} \text { and } X_{\mathrm{S}}=\frac{\eta}{\theta_{\mathrm{S}}} \text { and } p_{\mathrm{N}} \theta_{\mathrm{N}} X_{\mathrm{N}}+p_{\mathrm{S}} \theta_{\mathrm{S}} X_{\mathrm{S}} \leq \eta
$$

Figure 4 illustrates the numerical solutions based on empirical data. 
Figure 4. Solutions for use of the environment $\left(E_{\mathrm{N}}\right)$ as a function of per capita consumption $\left(X_{\mathrm{N}}\right)$ for OECD countries based on a concern for non-OECD countries: $\alpha_{N}=\alpha_{S}=0.25, \delta_{N}=0.73, \beta_{N}=\gamma_{N}=0.01, \beta_{S}=\gamma_{S}=0.005, \varepsilon$ $=\zeta=0.01$. The cluster of curves represents the relationship between $E_{N}$ and $X_{N}$ for four values of $\left(U_{F}-U_{C}\right) / U_{F}$, with the value decreasing from left to right. The thick decreasing curve to the right of this cluster represents the first-order conditions for Max $U$. The increasing straight line represents the border condition for $E F \leq E_{C}$. The horizontal straight line represents the first-order conditions for Max $W$. The highest, middle, and lowest dots are the representative OECD, world, and non-OECD individuals, respectively.

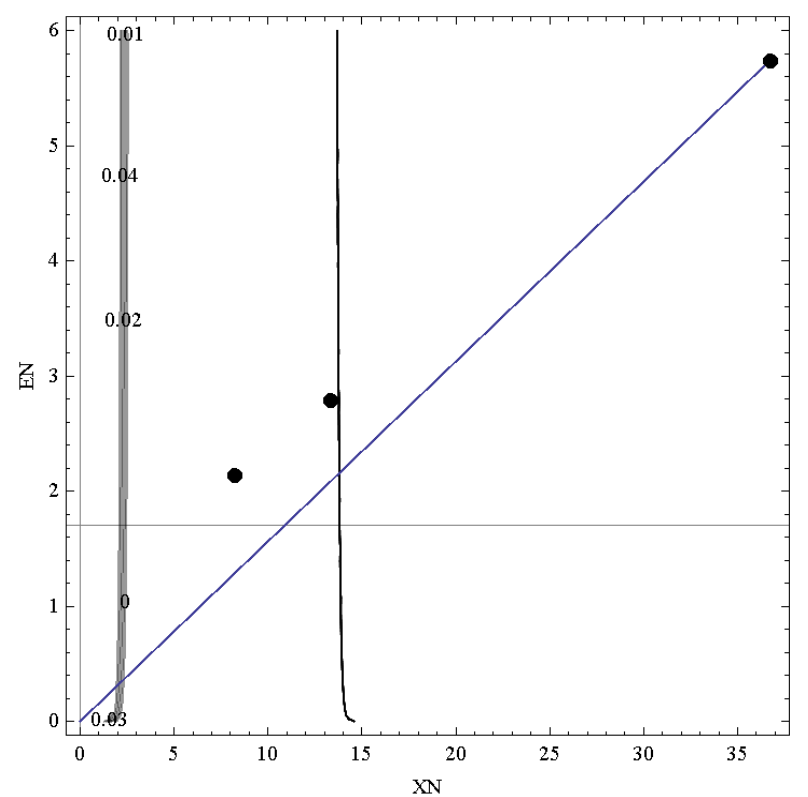

For a situation in which there is no concern for nature, future generations, and non-OECD countries (i.e., $\beta_{\mathrm{N}}=\gamma_{\mathrm{N}}=\delta_{\mathrm{N}}=0$ ) in OECD countries, and in which there is no concern for nature and future generations (i.e., $\beta_{S}=\gamma_{S}=0$ ) in non-OECD countries, and in which the current generation's aversion to inequality for the current non-OECD generation and the current generation's aversion to inequality for future generations are at their maximum (i.e., $\varepsilon=1$ so $p_{\mathrm{N}} U_{\mathrm{N}}=p_{\mathrm{S}} U_{\mathrm{S}}$ ) and at their minimum (i.e., $\zeta=0$ ), respectively, the analytical solutions for the four sustainability paradigms are as follows:

For weak sustainability (i.e., first-order conditions for Max $U$ and $U_{\mathrm{F}} \geq U_{\mathrm{C}}$ ):

$$
X_{\mathrm{N}}=X_{\mathrm{S}}^{\frac{\alpha \mathrm{SpS}}{\alpha \mathrm{NpN}}} \text { and } X_{\mathrm{N}} \leq \exp ^{\frac{U \mathrm{~F}}{\alpha \mathrm{N} p \mathrm{~N}}} X_{\mathrm{S}}^{-\frac{\alpha \mathrm{S} p \mathrm{~S}}{\alpha \mathrm{N} p \mathrm{~N}}}
$$

For a-growth (i.e., first-order conditions for Max $U$ and $E_{\mathrm{C}} \leq E_{\mathrm{F}}$ ):

$$
X_{\mathrm{N}}=X_{\mathrm{S}}{ }^{\frac{\alpha \mathrm{SpS}}{\alpha \mathrm{NpN}}} \text { and } p_{\mathrm{N}} \theta_{\mathrm{N}} X_{\mathrm{N}}+p_{\mathrm{S}} \theta_{\mathrm{S}} X_{\mathrm{S}} \leq \eta
$$

For de-growth (i.e., $U_{\mathrm{F}} \geq U C$ and $X C \leq X_{\mathrm{F}}$ ):

$$
X_{\mathrm{N}}=\exp ^{\frac{U \mathrm{~F}}{\alpha \mathrm{N} p \mathrm{~N}}} X_{\mathrm{S}}^{-\frac{\alpha \mathrm{S} p \mathrm{~S}}{\alpha \mathrm{N} p \mathrm{~N}}} \text { and } p_{\mathrm{N}} X_{\mathrm{N}}+p_{\mathrm{S}} X_{\mathrm{S}} \leq X_{\mathrm{F}}
$$

For strong sustainability (i.e., first-order conditions for $\operatorname{Max} W$ and $E_{\mathrm{C}} \leq E_{\mathrm{F}}$ ):

$$
X_{\mathrm{N}}=\frac{\eta}{\theta_{\mathrm{N}}} \text { and } X_{\mathrm{S}}=\frac{\eta}{\theta_{\mathrm{S}}} \text { and } p_{\mathrm{N}} \theta_{\mathrm{N}} X_{\mathrm{N}}+p_{\mathrm{S}} \theta_{\mathrm{S}} X_{\mathrm{S}} \leq \eta
$$

Figure 5 illustrates the numerical solutions based on empirical data. 
Figure 5. Solutions for use of the environment $\left(E_{\mathrm{N}}\right)$ as a function of per capita consumption $\left(X_{\mathrm{N}}\right)$ for $\mathrm{OECD}$ countries based on aversion to inequality for non-OECD countries: $\alpha_{N}=\alpha_{S}=0.25, \beta_{N}=\gamma_{N}=\delta_{N}=0.01, \beta_{S}=\gamma_{S}=$ $0.005, \varepsilon=0.99, \zeta=0.01$. The cluster of curves represents the relationship between $E_{N}$ and $X_{N}$ for four values of $\left(U_{\mathrm{F}}-U_{\mathrm{C}}\right) / U_{\mathrm{F}}$, with the value decreasing from left to right. The thick decreasing curve to the right of this cluster represents the first-order conditions for Max $U$. The increasing straight line represents the border condition for $E_{\mathrm{F}} \leq E_{\mathrm{C}}$. The horizontal straight line represents the first-order conditions for Max $W$. The highest, middle, and lowest dots are the representative OECD, world, and non-OECD individuals, respectively.

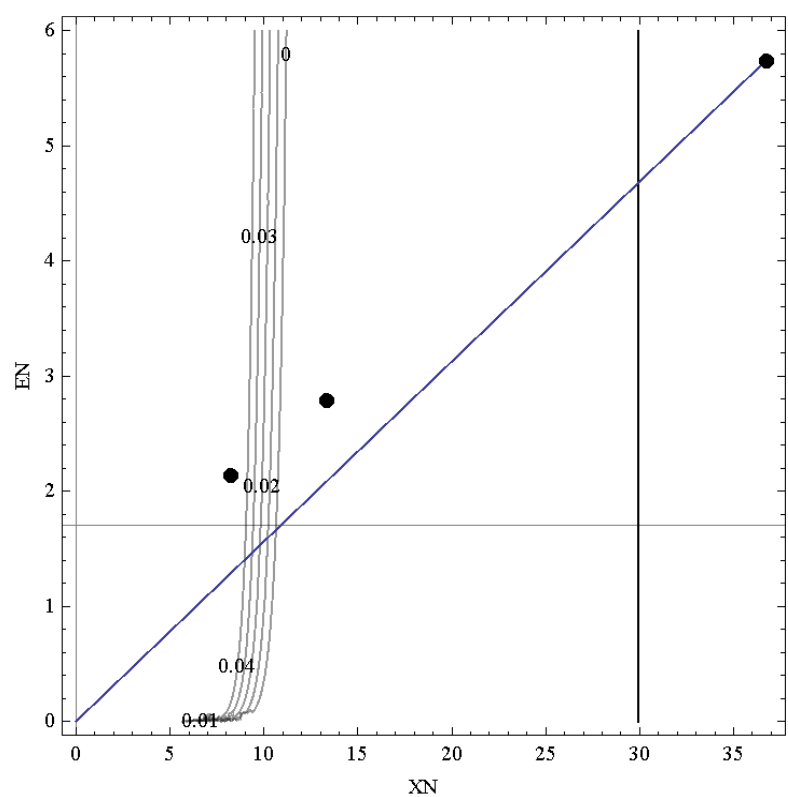

For a situation in which there is no concern for nature, future generations, and non-OECD countries (i.e., $\beta_{\mathrm{N}}=\gamma_{\mathrm{N}}=\delta_{\mathrm{N}}=0$ ) in OECD countries, and in which there is no concern for nature and future generations (i.e., $\beta_{S}=\gamma_{S}=0$ ) in non-OECD countries, and in which the current generation's aversion to inequality for the current non-OECD generation and the current generation's aversion to inequality for future generations are at their minimum (i.e., $\varepsilon=0$ ) and at their maximum (i.e., $\zeta=1$ so $U_{\mathrm{C}}=U_{\mathrm{F}}$ ), respectively, the analytical solutions for the four sustainability paradigms are as follows:

For weak sustainability (i.e., first-order conditions for Max $U$ and $U_{\mathrm{F}} \geq U_{\mathrm{C}}$ ):

$$
X_{\mathrm{N}}=\exp ^{\frac{U \mathrm{~F}}{\alpha \mathrm{N} p \mathrm{~N}}} X_{\mathrm{S}}^{-\frac{\alpha \mathrm{S} p \mathrm{~S}}{\alpha \mathrm{N} p \mathrm{~N}}} \text { and } X_{\mathrm{N}} \leq \exp ^{\frac{U \mathrm{~F}}{\alpha \mathrm{N} p \mathrm{~N}}} X_{\mathrm{S}}^{-\frac{\alpha \mathrm{S} p \mathrm{~S}}{\alpha \mathrm{N} p \mathrm{~N}}}
$$

For a-growth (i.e., first-order conditions for Max $U$ and $E_{\mathrm{C}} \leq E_{\mathrm{F}}$ ):

$$
X_{\mathrm{N}}=\exp ^{\frac{U \mathrm{~F}}{\alpha \mathrm{N} p}} X_{\mathrm{S}}^{-\frac{\alpha \mathrm{S} p \mathrm{~S}}{\alpha \mathrm{N} p \mathrm{~N}}} \text { and } p_{\mathrm{N}} \theta_{\mathrm{N}} X_{\mathrm{N}}+p_{\mathrm{S}} \theta_{\mathrm{S}} X_{\mathrm{S}} \leq \eta
$$

For de-growth (i.e., $U_{\mathrm{F}} \geq U_{\mathrm{C}}$ and $X_{\mathrm{C}} \leq X_{\mathrm{F}}$ ):

$$
X_{\mathrm{N}}=\exp ^{\frac{U \mathrm{~F}}{\alpha \mathrm{N} p \mathrm{~N}}} X_{\mathrm{S}}^{-\frac{\alpha \mathrm{S} p \mathrm{~S}}{\alpha \mathrm{N} p \mathrm{~N}}} \text { and } p_{\mathrm{N}} X_{\mathrm{N}}+p_{\mathrm{S}} X_{\mathrm{S}} \leq X_{\mathrm{F}}
$$

For strong sustainability (i.e., first-order conditions for Max $W$ and $E_{\mathrm{C}} \leq E_{\mathrm{F}}$ ):

$$
X_{\mathrm{N}}=\frac{\eta}{\theta_{\mathrm{N}}} \text { and } X_{\mathrm{S}}=\frac{\eta}{\theta_{\mathrm{S}}} \text { and } p_{\mathrm{N}} \theta_{\mathrm{N}} X_{\mathrm{N}}+p_{\mathrm{S}} \theta_{\mathrm{S}} X_{\mathrm{S}} \leq \eta
$$

Figure 6 illustrates the numerical solutions based on empirical data. 
Figure 6. Solutions for use of the environment $\left(E_{\mathrm{N}}\right)$ as a function of per capita consumption $\left(X_{\mathrm{N}}\right)$ for $\mathrm{OECD}$ countries based on aversion to inequality for future generations: $\alpha_{N}=\alpha S=0.25, \beta_{N}=\gamma_{N}=\delta_{N}=0.01, \beta S=\gamma_{S}=$ $0.005, \varepsilon=0.01, \zeta=0.99$. The cluster of curves represents the relationship between $E_{N}$ and $X_{N}$ for four values of $\left(U_{\mathrm{F}}-U_{\mathrm{C}}\right) / U_{\mathrm{F}}$, with the value decreasing from left to right. The thick decreasing curve to the right of this cluster represents the first-order conditions for $\operatorname{Max} U$. The increasing straight line represents the border condition for $E_{\mathrm{F}} \leq E_{\mathrm{C}}$. The horizontal straight line represents the first-order conditions for Max $W$. The highest, middle, and lowest dots are the representative OECD, world, and non-OECD individuals, respectively.

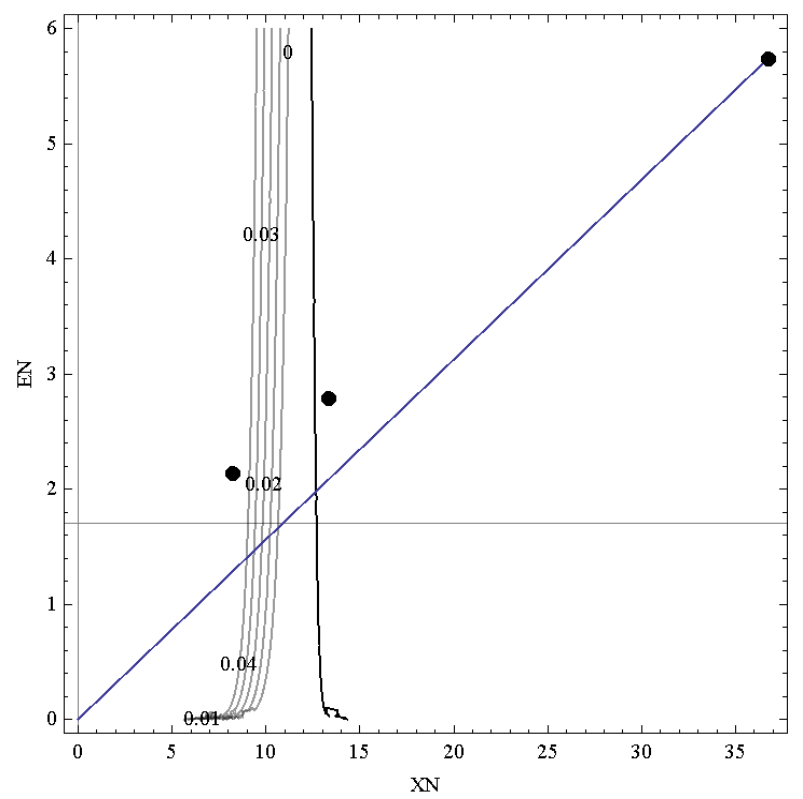

Figures 1 to 6 show that there are no solutions for weak sustainability, apart from the case with (extreme) responsibility for nature, whereas the other three sustainability paradigms always have solutions. Table 2 suggests that, with current preferences, a-growth and strong sustainability are slightly feasible, although these solutions are Gini-equitable for consumption, environment use, and welfare. Responsibility for nature makes all paradigms unfeasible. As expected, concern for future generations makes a-growth and strong sustainability feasible, and solutions are also MaxMinequitable for welfare; Gini-equitable for consumption, environment use, and welfare; and Paretoefficient. Surprisingly, a concern for non-OECD countries makes a-growth and strong sustainability moderately feasible, and solutions are Kaldor-Hicks efficient and Gini-equitable for consumption and environment use. Aversion to inequality for non-OECD countries makes a-growth and strong sustainability moderately and slightly feasible, respectively, with a reduction of welfare by $5 \%$ and $16 \%$ for OECD and non-OECD countries, respectively, for a-growth; and by $25 \%$ and $5 \%$ for strong sustainability, respectively. In addition, all strong sustainability solutions are Gini-equitable for consumption, environment use, and welfare, whereas a-growth is Kaldor-Hicks efficient. Aversion to inequality for future generations makes a-growth and strong sustainability slightly feasible, with a reduction of welfare by $23 \%$ and $6 \%$ for OECD and non-OECD countries, respectively, for a-growth; and by $25 \%$ and $5 \%$ for strong sustainability, respectively. In addition, all solutions are Gini-equitable for consumption, environment use, and welfare. 
Table 2. Feasibility (green $=$ feasible, yellow $=$ moderately feasible, red $=$ slightly feasible, white $=$ unfeasible , and levels and percent changes in consumption $(X)$, environment use $(E)$, and welfare $(U)$. Efficiency $\left({ }^{\circ}=\right.$ Pareto; underlined $=$ Kaldor-Hicks) and equality $($ bold $=$ MaxMin; italics $=$ Gini $)$. Current preferences $(\mathbf{C P})=$

sustainability with today's preferences; responsibility for nature exists if $\beta_{N}=\beta_{S}=0.73$; concern for future generations exists if $\gamma_{N}=\gamma_{S}=0.73$; concern for non-OECD countries exists if $\delta_{N}=0.73$; aversion to inequality for non-OECD countries exists if $\varepsilon=0.99$; aversion to inequality for future generations exists if $\zeta=0.99$.

Sustainability paradigms: WS, weak sustainability; AG, a-growth; DG, de-growth; SS, strong sustainability.

\begin{tabular}{|c|c|c|c|c|c|c|c|c|c|c|c|c|}
\hline & \multicolumn{2}{|c|}{$\mathrm{CP}$} & \multicolumn{2}{|c|}{$\begin{array}{l}\text { Responsibility } \\
\text { for nature }\end{array}$} & \multicolumn{2}{|c|}{$\begin{array}{l}\text { Concern for } \\
\text { future } \\
\text { generations }\end{array}$} & \multicolumn{2}{|c|}{$\begin{array}{l}\text { Concern for non- } \\
\text { OECD countries }\end{array}$} & \multicolumn{2}{|c|}{$\begin{array}{l}\text { Aversion to } \\
\text { inequality for non- } \\
\text { OECD countries }\end{array}$} & \multicolumn{2}{|c|}{$\begin{array}{c}\text { Aversion to } \\
\text { inequality for } \\
\text { future generations }\end{array}$} \\
\hline & Level & \begin{tabular}{|c|} 
Change \\
$\%$
\end{tabular} & Level & $\begin{array}{c}\text { Change } \\
\%\end{array}$ & Level & $\begin{array}{c}\text { Change } \\
\%\end{array}$ & Level & $\begin{array}{c}\text { Change } \\
\%\end{array}$ & Level & $\begin{array}{c}\text { Change } \\
\%\end{array}$ & Level & $\begin{array}{c}\text { Change } \\
\%\end{array}$ \\
\hline \multicolumn{13}{|l|}{ WS } \\
\hline$X_{\mathrm{N}}$ & & & 17.50 & -52 & & & & & & & & \\
\hline$E_{\mathrm{N}}$ & & & 1.15 & -80 & & & & & & & & \\
\hline$U_{\mathrm{N}}$ & & & 1.86 & -24 & & & & & & & & \\
\hline$X_{\mathrm{S}}$ & & & 5.64 & -31 & & & & & & & & \\
\hline$E_{\mathrm{S}}$ & & & 1.82 & -15 & & & & & & & & \\
\hline$U_{\mathrm{S}}$ & & & 1.00 & -41 & & & & & & & & \\
\hline \multicolumn{13}{|l|}{ AG } \\
\hline$X_{\mathrm{N}}$ & 12.67 & -66 & 11.77 & -68 & 12.68 & -65 & 13.79 & -62 & 29.90 & -19 & 12.50 & -66 \\
\hline$E_{\mathrm{N}}$ & 1.98 & -65 & 1.84 & -68 & 1.98 & -65 & 2.16 & -62 & 4.67 & -19 & 1.95 & -66 \\
\hline$U_{\mathrm{N}}$ & 1.89 & -22 & 1.20 & -51 & ${\underline{2.92^{\circ}}}^{\circ}$ & 20 & 2.68 & 10 & 2.32 & -5 & 1.89 & -23 \\
\hline$X_{\mathrm{S}}$ & 6.28 & -24 & 6.40 & -22 & 6.28 & -24 & 6.13 & -25 & 4.01 & -51 & 6.30 & -23 \\
\hline$E_{\mathrm{S}}$ & 1.64 & -23 & 1.67 & -22 & 1.64 & -23 & 1.60 & -25 & 1.05 & -51 & 1.64 & -23 \\
\hline$U_{\mathrm{S}}$ & 1.58 & -6 & 1.10 & -35 & ${\underline{2.44^{\circ}}}^{\circ}$ & 44 & $\underline{1.57}$ & -7 & $\underline{1.42}$ & -16 & 1.59 & -6 \\
\hline \multicolumn{13}{|l|}{ DG } \\
\hline$X_{\mathrm{N}}$ & 10.63 & -71 & 4.91 & -87 & 1.74 & -95 & 2.31 & -94 & 10.63 & -71 & 10.63 & -71 \\
\hline$E_{\mathrm{N}}$ & 1.66 & -71 & 0.77 & -87 & 0.27 & -95 & 0.36 & -94 & 1.66 & -71 & 1.66 & -71 \\
\hline$U_{\mathrm{N}}$ & 1.82 & -26 & 1.82 & -26 & 1.82 & -26 & 1.82 & -26 & 1.82 & -26 & 1.82 & -26 \\
\hline$X_{\mathrm{S}}$ & 6.55 & -20 & 7.30 & -11 & 7.71 & -6 & 7.64 & -7 & 6.55 & -20 & 6.55 & -20 \\
\hline$E_{\mathrm{S}}$ & 1.71 & -20 & 1.90 & -11 & 2.01 & -6 & 1.99 & -7 & 1.71 & -20 & 1.71 & -20 \\
\hline$U_{\mathrm{S}}$ & 1.60 & -5 & 1.03 & -39 & 2.57 & 52 & 1.66 & -2 & 1.60 & -5 & 1.60 & -5 \\
\hline \multicolumn{13}{|l|}{ SS } \\
\hline$X_{\mathrm{N}}$ & 10.87 & -70 & 10.87 & -70 & 10.87 & -70 & 10.87 & -70 & 10.87 & -70 & 10.87 & -70 \\
\hline$E_{\mathrm{N}}$ & 1.70 & -70 & 1.70 & -70 & 1.70 & -70 & 1.70 & -70 & 1.70 & -70 & 1.70 & -70 \\
\hline$U_{\mathrm{N}}$ & 1.83 & -25 & 1.24 & -49 & ${\underline{2.82^{\circ}}}^{\circ}$ & 15 & $\underline{2.56}$ & 5 & 1.83 & -25 & 1.83 & -25 \\
\hline$X_{\mathrm{S}}$ & 6.51 & -21 & 6.51 & -21 & 6.51 & -21 & 6.51 & -21 & 6.51 & -21 & 6.51 & -21 \\
\hline$E_{\mathrm{S}}$ & 1.70 & -21 & 1.70 & -21 & 1.70 & -21 & 1.70 & -21 & 1.70 & -21 & 1.70 & -21 \\
\hline$U_{\mathrm{S}}$ & 1.60 & -5 & 1.09 & -36 & $2.46^{\circ}$ & 46 & $\underline{1.60}$ & -5 & 1.60 & -5 & 1.60 & -5 \\
\hline
\end{tabular}

\subsection{Interior solutions}

In this section, I search for feasible solutions arising from changes in all preference parameters related to a sense of concern or responsibility, for alternative values of the aversion to inequality parameters, by measuring feasibility in terms of acceptable welfare losses in OECD and non-OECD countries.

Figures 7 to 9 show that there are no solutions for de-growth, whereas the other three sustainability paradigms have some solutions. Table 3 shows that weak sustainability is slightly feasible with small (and moderate) aversion to inequality: solutions are Gini-equitable for consumption, environment use, and welfare and Kaldor-Hicks efficient and Gini-equitable for consumption and welfare. A-growth is slightly feasible with all levels of aversion to inequality, although the sustainability burden is larger for people of the non-OECD countries at larger values of $\varepsilon$ and $\zeta$; solutions are Gini-equitable for consumption, environment use, and welfare with small and moderate $\varepsilon$ and $\zeta$, and Kaldor-Hicks efficient with moderate and large $\varepsilon$ and $\zeta$. Strong sustainability is always slightly feasible, regardless of $\varepsilon$ and $\zeta$; all solutions are Gini-equitable for consumption, environment use, and welfare. 
Figure 7. Solutions for use of the environment $\left(E_{N}\right)$ as a function of per capita consumption $\left(X_{N}\right)$ for OECD countries based on all preference parameters related to a sense of concern or responsibility and small aversion to inequality: $\alpha_{N}=\beta_{N}=\gamma_{N}=\delta_{N}=0.25, \alpha_{S}=\beta_{S}=\gamma_{S}=0.25, \varepsilon=\zeta=0.01$. The cluster of curves represents the relationship between $E_{N}$ and $X_{N}$ for four values of $\left(U_{F}-U_{C}\right) / U_{F}$, with the value decreasing from left to right. The thick decreasing curve represents the first-order conditions for Max $U$. The increasing straight line represents the border condition for $E_{\mathrm{F}} \leq E_{\mathrm{C}}$. The horizontal straight line represents the first-order conditions for Max $W$.

The highest, middle, and lowest dots are the representative OECD, world, and non-OECD individuals.

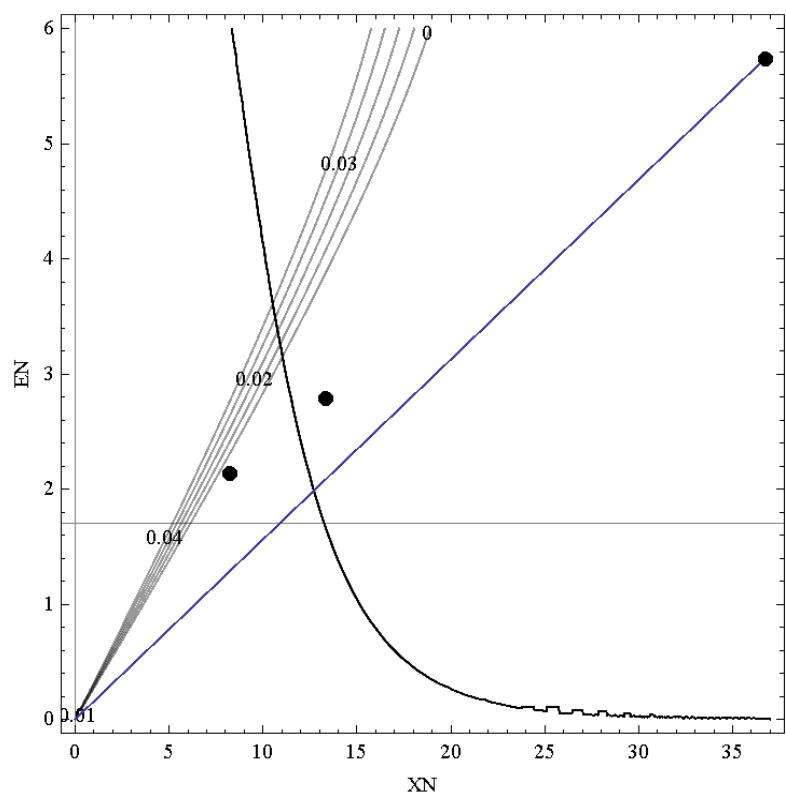

Figure 8. Solutions for use of the environment $\left(E_{\mathrm{N}}\right)$ as a function of per capita consumption $\left(X_{\mathrm{N}}\right)$ for OECD countries based on all preference parameters related to a sense of concern or responsibility and medium aversion to inequality: $\alpha_{N}=\beta_{N}=\gamma_{N}=\delta_{N}=0.25, \alpha_{S}=\beta_{S}=\gamma_{S}=0.25, \varepsilon=\zeta=0.50$. The cluster of curves represents the relationship between $E_{N}$ and $X_{N}$ for four values of $\left(U_{F}-U_{C}\right) / U_{F}$, with the value decreasing from left to right. The thick decreasing curve represents the first-order conditions for Max $U$. The increasing straight line represents the border condition for $E_{\mathrm{F}} \leq E_{\mathrm{C}}$. The horizontal straight line represents the first-order conditions for Max $W$.

The highest, middle, and lowest dots are the representative OECD, world, and non-OECD individuals.

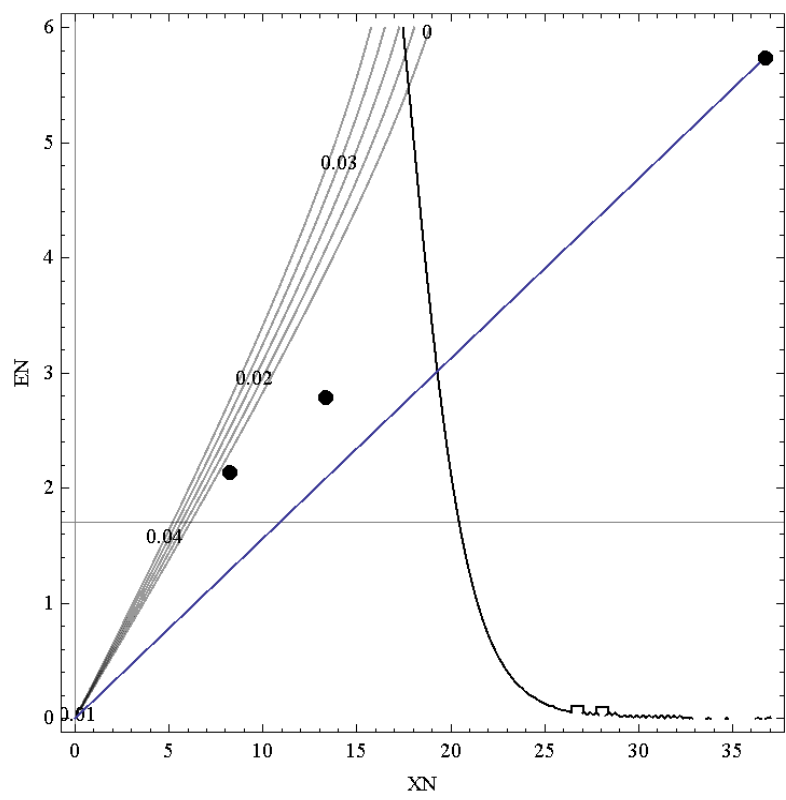


Figure 9. Solutions for use of the environment $\left(E_{\mathrm{N}}\right)$ as a function of per capita consumption $\left(X_{\mathrm{N}}\right)$ for $\mathrm{OECD}$ countries based on all preference parameters related to a sense of concern or responsibility and large aversion to inequality: $\alpha_{N}=\beta_{N}=\gamma_{N}=\delta_{N}=0.25, \alpha_{S}=\beta_{S}=\gamma_{S}=0.25, \varepsilon=\zeta=0.99$. The cluster of curves represents the relationship between $E_{N}$ and $X_{N}$ for four values of $\left(U_{F}-U_{C}\right) / U_{F}$, with the value decreasing from left to right. The thick decreasing curve represents the first-order conditions for Max $U$. The increasing straight line represents the border condition for $E_{\mathrm{F}} \leq E_{\mathrm{C}}$. The horizontal straight line represents the first-order conditions for Max $W$.

The highest, middle, and lowest dots are the representative OECD, world, and non-OECD individuals.

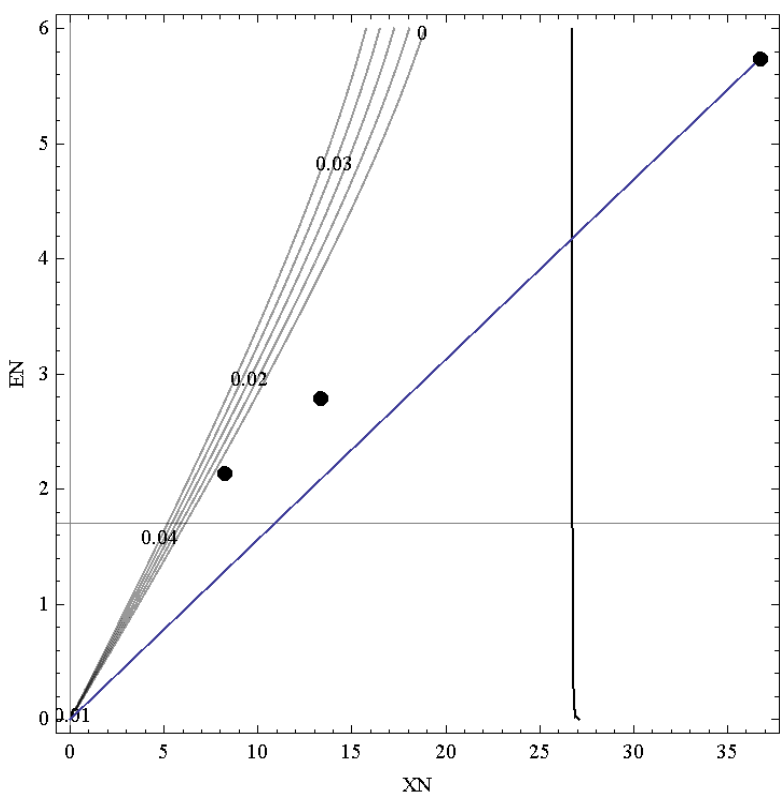

Table 3. Feasibility levels (yellow = moderately feasible, red = slightly feasible, white $=$ unfeasible), and percent changes in consumption $(X)$, environment use $(E)$ and welfare $(U)$. Efficiency (underlined = Kaldor-Hicks) and equity (italics $=$ Gini). Small, medium, and large aversion to inequality for non-OECD countries and future generations exist if $\varepsilon=\zeta=0.01, \varepsilon=\zeta=0.50$, and $\varepsilon=\zeta=0.99$, respectively. Sustainability paradigms: WS, weak sustainability; AG, a-growth; SS, strong sustainability. De-growth is not shown because there were no solutions (see Figures 7-9).

\begin{tabular}{|c|c|c|c|c|c|c|}
\hline & \multicolumn{2}{|c|}{ Small inequality aversion } & \multicolumn{2}{l|}{ Medium inequality aversion } & \multicolumn{2}{l|}{ Large inequality aversion } \\
\hline & Level & Change (\%) & Level & Change (\%) & Level & Change (\%) \\
\hline $\mathbf{W S}$ & & & & & & \\
\hline$X_{\mathrm{N}}$ & 11.00 & -70 & 17.50 & -52 & & \\
\hline$E_{\mathrm{N}}$ & 3.15 & -45 & 5.40 & -6 & & \\
\hline$U_{\mathrm{N}}$ & 1.82 & -25 & $\underline{2.03}$ & -17 & & \\
\hline$X_{\mathrm{S}}$ & 6.50 & -21 & 5.64 & -31 & & \\
\hline$E_{\mathrm{S}}$ & 1.38 & -35 & 0.89 & -59 & & \\
\hline$U_{\mathrm{S}}$ & 1.60 & -5 & $\underline{1.55}$ & -8 & & \\
\hline $\mathbf{A G}$ & & & & & & \\
\hline$X_{\mathrm{N}}$ & 12.70 & -65 & 19.27 & -48 & 26.70 & -27 \\
\hline$E_{\mathrm{N}}$ & 1.99 & -65 & 3.01 & -48 & 4.17 & -27 \\
\hline$U_{\mathrm{N}}$ & 1.89 & -22 & $\underline{2.09}$ & -14 & 2.26 & -7 \\
\hline$X_{\mathrm{S}}$ & 6.27 & -24 & 5.41 & -34 & 4.43 & -46 \\
\hline$E_{\mathrm{S}}$ & 1.64 & -23 & 1.41 & -34 & 1.16 & -46 \\
\hline$U_{\mathrm{S}}$ & 1.58 & -6 & $\underline{1.53}$ & -10 & 1.45 & -14 \\
\hline $\mathbf{S S}$ & & & & & & \\
\hline$X_{\mathrm{N}}$ & 10.87 & -70 & 10.87 & -70 & 10.87 & -70 \\
\hline$E_{\mathrm{N}}$ & 1.70 & -70 & 1.70 & -70 & 1.70 & -70 \\
\hline$U_{\mathrm{N}}$ & 1.83 & -25 & 1.83 & -25 & 1.83 & -25 \\
\hline$X_{\mathrm{S}}$ & 6.51 & -21 & 6.51 & -21 & 6.51 & -21 \\
\hline$E_{\mathrm{S}}$ & 1.70 & -21 & 1.70 & -21 & 1.70 & -21 \\
\hline$U_{\mathrm{S}}$ & 1.60 & -5 & 1.60 & -5 & 1.60 & -5 \\
\hline & & & & & \\
\hline
\end{tabular}




\subsection{Sensitivity analyses}

In this section, I search for feasible solutions at current preferences but in three different contexts: improved technology, decreased population, and modified consumption. Since the welfare of future generations is affected by all three context changes, I have modified the reference values accordingly: $U_{\mathrm{F}}=1.81$ becomes $U_{\mathrm{F}}=1.95,1.92$, and 1.56 in contexts with changes in technology $(\theta)$, population $(\eta)$, and consumption $(\alpha)$, respectively, whereas $X_{\mathrm{F}}=10.876$ becomes $X_{\mathrm{F}}=14.502$ and 13.595 in contexts with changes in $\theta$ and $\eta$, respectively.

Figures 10 to 12 show that there are no solutions for weak sustainability, whereas the other three sustainability paradigms always have solutions. Table 4 shows that improved technology makes agrowth, de-growth, and strong sustainability slightly feasible. In addition, the solutions are KaldorHicks efficient; Gini-equitable for consumption, environment use, and welfare; and MaxMinequitable for consumption and welfare. Decreased population makes a-growth, de-growth, and strong sustainability slightly feasible. In addition, the solutions are Kaldor-Hicks efficient and Giniequitable for consumption, environment use, and welfare. Modified consumption makes a-growth slightly feasible and de-growth and strong sustainability moderately feasible. In addition, all solutions are Gini-equitable for consumption, environment use, and welfare.

Figure 10. Solutions for use of the environment $\left(E_{N}\right)$ as a function of per capita consumption $\left(X_{N}\right)$ for OECD countries based on improved technology (i.e., increased environmental efficiency, $\theta$ ): $\alpha_{N}=\alpha_{S}=0.25, \beta_{N}=\gamma_{N}=\delta_{N}$ $=0.01, \beta_{S}=\gamma_{S}=0.005, \varepsilon=\zeta=0.01, \eta=1.7, \theta_{N}=0.1172, \theta_{S}=0.1957$. The cluster of curves represents the relationship between $E_{N}$ and $X_{N}$ for four values of $\left(U_{F}-U_{C}\right) / U_{F}$, with the value decreasing from left to right. The thick decreasing curve represents the first-order conditions for Max $U$. The increasing straight line represents the border condition for $E_{\mathrm{F}} \leq E_{\mathrm{C}}$. The horizontal straight line represents the first-order conditions for Max $W$.

The highest, middle, and lowest dots are the representative OECD, world, and non-OECD individuals, respectively.

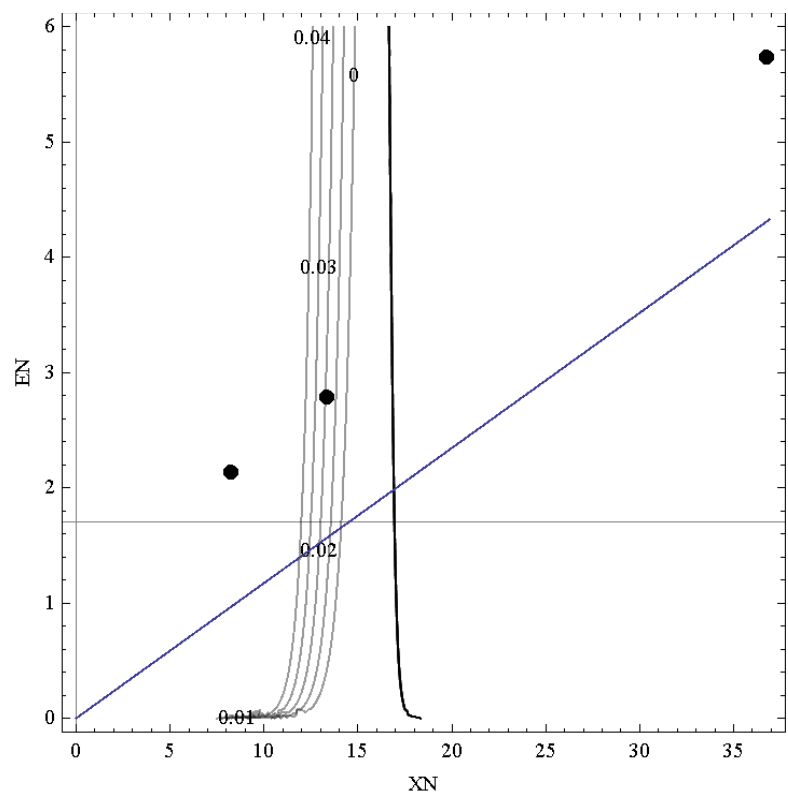


Figure 11. Solutions for use of the environment $\left(E_{\mathrm{N}}\right)$ as a function of per capita consumption $\left(X_{\mathrm{N}}\right)$ for $\mathrm{OECD}$ countries based on reduced world population $(\eta): \alpha_{N}=\alpha_{S}=0.25, \beta_{N}=\gamma_{N}=\delta_{N}=0.01, \beta_{S}=\gamma_{S}=0.005, \varepsilon=\zeta=0.01$, $\eta=2.125, \theta_{\mathrm{N}}=0.1563, \theta_{\mathrm{S}}=0.2610$. Numerical values on each curve represent $\left(U_{\mathrm{F}}-U C\right) / U_{\mathrm{F}}$. The cluster of curves represents the relationship between $E_{N}$ and $X_{N}$ for four values of $\left(U_{F}-U_{C}\right) / U_{F}$, with the value decreasing from left to right. The thick decreasing curve represents the first-order conditions for Max $U$. The increasing straight line represents the border condition for $E_{\mathrm{F}} \leq E_{\mathrm{C}}$. The horizontal straight line represents the first-order conditions for Max $W$. The highest, middle, and lowest dots are the representative OECD, world, and non-OECD individuals, respectively.

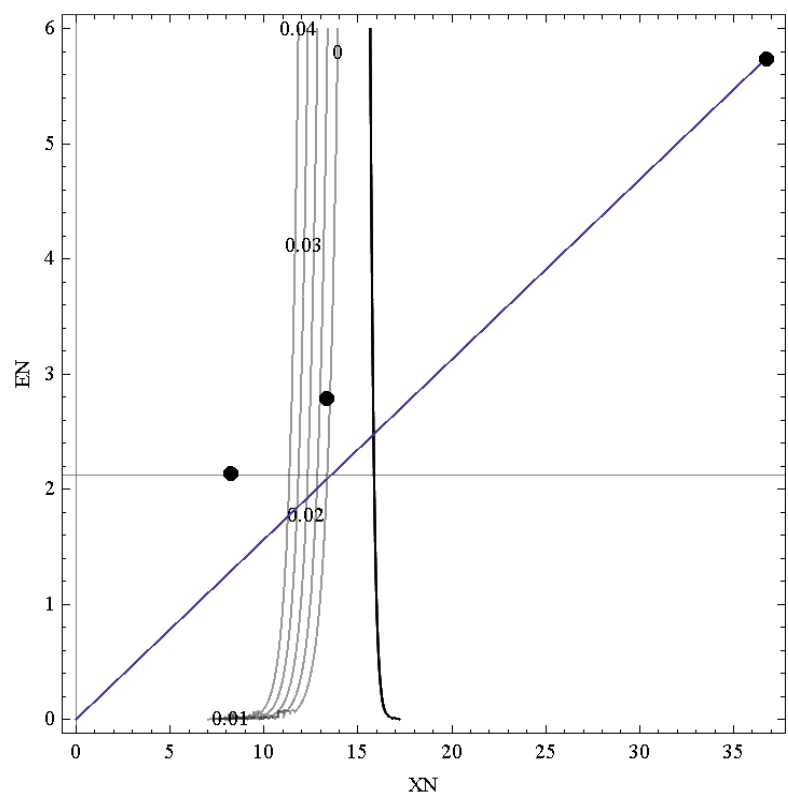

Figure 12. Solutions for use of the environment $\left(E_{\mathrm{N}}\right)$ as a function of per capita consumption $\left(X_{\mathrm{N}}\right)$ for $\mathrm{OECD}$ countries based on changed consumption preferences $(\alpha): \alpha_{N}=\alpha_{S}=0.1875, \beta_{N}=\gamma_{N}=\delta_{N}=0.01, \beta_{S}=\gamma_{S}=0.005, \varepsilon$ $=\zeta=0.01, \eta=1.7, \theta_{\mathrm{N}}=0.1563, \theta_{\mathrm{S}}=0.2610$. The cluster of curves represents the relationship between $E_{\mathrm{N}}$ and $X_{\mathrm{N}}$

for four values of $\left(U_{\mathrm{F}}-U_{\mathrm{C}}\right) / U_{\mathrm{F}}$, with the value decreasing from left to right. The thick decreasing curve represents the first-order conditions for Max $U$. The increasing straight line represents the border condition for $E_{\mathrm{F}} \leq E_{\mathrm{C}}$. The horizontal straight line represents the first-order conditions for Max $W$. The highest, middle, and lowest dots are the representative OECD, world, and non-OECD individuals, respectively.

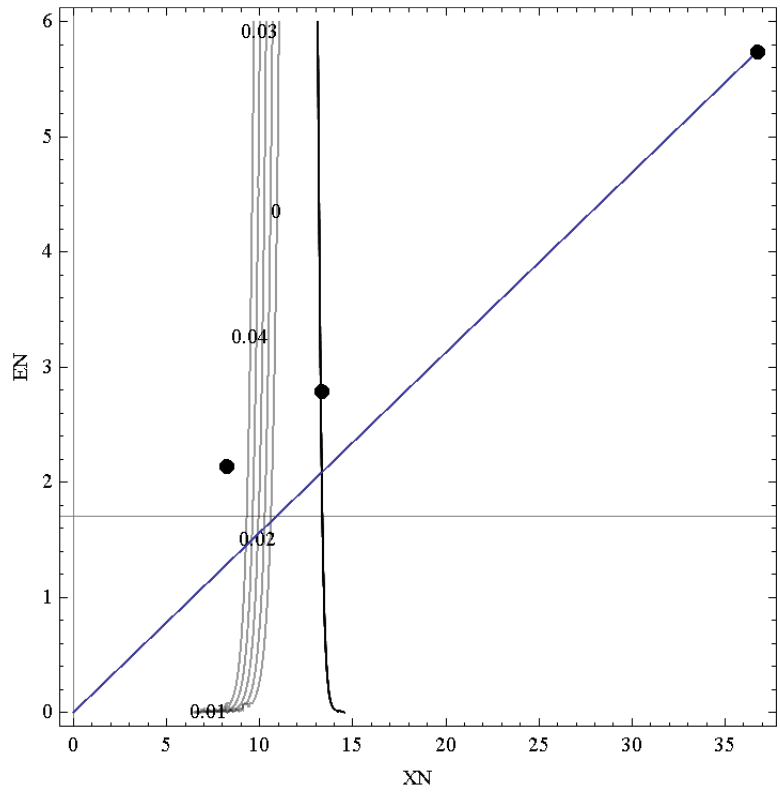


Table 4. Feasibility levels $($ green $=$ feasible, yellow $=$ moderately feasible, red $=$ slightly feasible, white $=$ unfeasible), and percent changes in consumption $(X)$, environment use $(E)$ and welfare $(U)$. Efficiency (underlined $=$ Kaldor-Hicks) and equality (bold = MaxMin; italic $=$ Gini). Improved technology exists if $\theta_{\mathrm{N}}=$ 0.1172 and $\theta_{S}=0.1957$; decreased population exists if $\eta=2.125$; modified consumption exists if $\alpha_{N}=\alpha_{S}=0.1875$. Sustainability paradigms: AG, a-growth; DG, de-growth; SS, strong sustainability. Weak sustainability is not shown because there were no feasible solutions (see Figures 10-12).

\begin{tabular}{|c|c|c|c|c|c|c|}
\hline & \multicolumn{2}{|c|}{ Improved technology } & \multicolumn{2}{c|}{ Decreased population } & \multicolumn{2}{c|}{ Modified consumption } \\
\hline & Level & Change (\%) & Level & Change(\%) & Level & Change(\%) \\
\hline $\mathbf{A G}$ & & & & & & \\
\hline$X_{\mathrm{N}}$ & $\mathbf{1 6 . 9 1}$ & -54 & 15.83 & -57 & 13.32 & -64 \\
\hline$E_{\mathrm{N}}$ & 1.98 & -65 & 2.47 & -57 & 2.08 & -64 \\
\hline$U_{\mathrm{N}}$ & $\mathbf{2 . 0 4}$ & -16 & $\underline{2.00}$ & -18 & $\underline{\mathbf{2 . 2 6}}$ & -7 \\
\hline$X_{\mathrm{S}}$ & $\mathbf{8 . 3 7}$ & 2 & 7.85 & -4 & 6.19 & -25 \\
\hline$E_{\mathrm{S}}$ & 1.64 & -23 & 2.05 & -4 & 1.62 & -24 \\
\hline$U_{\mathrm{S}}$ & $\mathbf{1 . 7 0}$ & 1 & $\underline{1.67}$ & -1 & $\underline{\mathbf{1 . 7 7}}$ & 5 \\
\hline $\mathbf{D G}$ & & & & & & \\
\hline$X_{\mathrm{N}}$ & $\mathbf{1 4 . 0 9}$ & -62 & 13.35 & -64 & 10.59 & -71 \\
\hline$E_{\mathrm{N}}$ & 1.65 & -71 & 2.09 & -64 & 1.66 & -71 \\
\hline$U_{\mathrm{N}}$ & $\underline{\mathbf{1 . 9 5}}$ & -20 & $\underline{1.92}$ & -21 & $\underline{\mathbf{2 . 1 1}}$ & -14 \\
\hline$X_{\mathrm{S}}$ & $\mathbf{8 . 7 4}$ & 6 & 8.17 & -1 & 6.55 & -20 \\
\hline$E_{\mathrm{S}}$ & 1.71 & -20 & 2.13 & 0 & 1.71 & -20 \\
\hline$U_{\mathrm{S}}$ & $\underline{\mathbf{1 . 7 2}}$ & 2 & $\underline{\mathbf{1 . 6 9}}$ & 0 & $\underline{\mathbf{1 . 8 0}}$ & 7 \\
\hline $\mathbf{S S}$ & & & & & & \\
\hline$X_{\mathrm{N}}$ & $\mathbf{1 4 . 5 0}$ & -61 & 13.59 & -63 & 10.87 & -70 \\
\hline$E_{\mathrm{N}}$ & 1.70 & -70 & 2.12 & -63 & 1.70 & -70 \\
\hline$U_{\mathrm{N}}$ & $\underline{\mathbf{1 . 9 6}}$ & -19 & $\underline{1.93}$ & -21 & $\underline{\mathbf{2 . 1 2}}$ & -13 \\
\hline$X_{\mathrm{S}}$ & $\mathbf{8 . 6 8}$ & 6 & 8.14 & -1 & 6.51 & -21 \\
\hline$E_{\mathrm{S}}$ & 1.70 & -21 & 2.13 & -1 & 1.70 & -21 \\
\hline$U_{\mathrm{S}}$ & $\underline{\mathbf{1 . 7 2}}$ & 2 & $\underline{1.69}$ & 0 & $\underline{\mathbf{1 . 8 0}}$ & 6 \\
\hline
\end{tabular}

\section{Discussion}

From a positive perspective, the insights about sustainability paradigms and value changes can be summarised as follows (Table 5). By considering the effects of modified consumption, decreased population and improved technology, the ordering of sustainability paradigms is a-growth $>$ strong sustainability $>$ de-growth $>$ weak sustainability. Note that de-growth is unfeasible, by disregarding these effects. A sense of responsibility for nature $\beta$ never produced feasible solution, whereas the ordering of other value changes is $\gamma>\delta>\varepsilon>\zeta$. Note that all senses of responsibility combined with medium inequality aversions are ranked II, like the effect of an improved technology $\theta$; all senses of responsibility combined with small or large inequality aversions are ranked III, like the effects of modified consumption $\alpha$ and decreased population $\eta$. Thus, $\gamma>\delta=(\beta, \gamma, \delta)$ with medium $(\varepsilon, \zeta)=\theta>$ $\varepsilon=\alpha=\eta=(\beta, \gamma, \delta)$ with small $(\varepsilon, \zeta)=(\beta, \gamma, \delta)$ with large $(\varepsilon, \zeta)>$ current preferences $=\zeta$.

Table 5. Feasibility. CP = current preferences, Fea = feasible, Mod = moderately feasible, Sli = slightly feasible, blank = unfeasible. Sustainability paradigms: WS, weak sustainability; AG, a-growth; DG, de-growth; SS, strong sustainability.

\begin{tabular}{|c|c|c|c|c|c|c|c|c|c|c|c|c|c|}
\hline & $\mathrm{CP}$ & $\beta$ & $\gamma$ & $\delta$ & $\varepsilon$ & $\zeta$ & $\begin{array}{c}\beta, \gamma, \delta \text { with } \\
\text { small } \varepsilon, \zeta\end{array}$ & $\begin{array}{c}\beta, \gamma, \delta \text { with } \\
\text { medium } \varepsilon, \zeta\end{array}$ & $\begin{array}{c}\beta, \gamma, \delta \text { with } \\
\text { large } \varepsilon, \zeta\end{array}$ & $\alpha$ & $\eta$ & $\theta$ & Rank \\
\hline WS & & & & & & Sli & Sli & & & & & IV \\
\hline AG & Sli & & Fea & Mod & Mod & Sli & Sli & Mod & Mod & Sli & Sli & Mod & I \\
\hline DG & & & & & & & & & & Sli & Sli & Sli & III \\
\hline SS & Sli & Fea & Mod & Sli & Sli & Sli & Sli & Sli & Sli & Sli & Sli & II \\
\hline Rank & & & I & II & III & IV & III & II & III & III & III & II & \\
\hline
\end{tabular}


From a normative perspective, in terms of internal consistency, the insights about sustainability paradigms and value changes can be summarised as follows (Table 6). A case consistent with weak sustainability (i.e., Kaldor-Hicks efficient with small $\varepsilon$ and $\zeta$ ) has no solutions. A case consistent with a-growth (i.e., Gini-equitable for welfare with small $\varepsilon$ and $\zeta$ ) allows a choice between $\gamma, \delta$, all senses of responsibility combined, and all context changes, although $\gamma$ is more feasible. A case consistent with de-growth (i.e., MaxMin-equitable for welfare for any $\varepsilon$ and $\zeta$ ) must rely on context changes. A case consistent with strong sustainability (i.e., Gini-equitable for consumption and environment use for any $\varepsilon$ and $\zeta$ ) allows a choice between $\gamma, \varepsilon$, and $\zeta$, all senses of responsibility combined, and all context changes, although $\gamma$ is more feasible. In summary, by considering the effects of modified consumption, decreased population and improved technology, the ordering of sustainability paradigms is strong sustainability $>$ a-growth $>$ de-growth, whereas weak sustainability was never internally consistent. Note that de-growth is internally inconsistent, by disregarding these effects. Although modified consumption, decreased population and improved technology are ranked better than a sense of responsibility for future generations (i.e., $\alpha=\eta=\theta>$ $\gamma)$, the ordering of value changes is $\gamma=(\beta, \gamma, \delta)$ with all $(\varepsilon, \zeta)>\delta=\varepsilon=\zeta$.

Table 6. Internal consistency. Yes = internally consistent, blank = internally inconsistent. $\mathbf{C P}=$ current preferences. Sustainability paradigms: WS, weak sustainability; AG, a-growth; DG, de-growth; SS, strong sustainability.

\begin{tabular}{|c|c|c|c|c|c|c|c|c|c|c|c|c|c|}
\hline & CP & $\beta$ & $\gamma$ & $\delta$ & $\varepsilon$ & $\zeta$ & $\begin{array}{c}\beta, \gamma, \delta \text { with } \\
\text { small } \varepsilon, \zeta\end{array}$ & $\begin{array}{c}\beta, \gamma, \delta \text { with } \\
\text { medium } \varepsilon, \zeta\end{array}$ & $\begin{array}{c}\beta, \gamma, \delta \text { with } \\
\text { large } \varepsilon, \zeta\end{array}$ & $\alpha$ & $\eta$ & $\theta$ & Rank \\
\hline WS & & & & & & & & & & & & IV \\
\hline AG & & & Yes & Yes & & & Yes & Yes & Yes & Yes & Yes & Yes & II \\
\hline DG & & & & & & & & & & Yes & Yes & Yes & III \\
\hline SS & & & Yes & & Yes & Yes & Yes & Yes & Yes & Yes & Yes & Yes & I \\
\hline Rank & & & II & III & IV & IV & II & II & II & I & I & I & \\
\hline
\end{tabular}

From a normative perspective, in terms of consistency with equity approaches, the insights about sustainability paradigms and value changes can be summarised as follows (Table 7). A case consistent with the utilitarian approach (here, Harsanyi; Kaldor-Hicks efficient with small $\varepsilon$ and $\zeta$ ) could be a-growth with a change in $\gamma$ or $\delta$, or strong sustainability with a change in $\gamma$ or $\delta$.

With a focus on consumption or environment (here Dworkin; Gini-equitable for consumption or environment use), a case consistent with the egalitarian approach could be weak sustainability with all senses of responsibility combined and small or medium aversion to inequality; it could be agrowth with current preferences, a change in $\gamma, \delta$, or $\zeta$, all preference parameters related to a sense of concern or responsibility with small or medium aversion to inequality, and all context changes; it could be de-growth with all context changes; or it could be strong sustainability with current preferences, a change in $\gamma, \delta$, $\varepsilon$, or $\zeta$, all preference parameters related to a sense of concern or responsibility with all aversion to inequality, and all context changes.

With a focus on welfare (here, Arneson; Gini-equitable in welfare), a case consistent with the egalitarian approach could be weak sustainability with all preference parameters related to a sense of concern or responsibility and small or medium aversion to inequality; it could be a-growth with current preferences, a change in $\gamma$ or $\zeta$, all preference parameters related to a sense of concern or responsibility with all levels of aversion, and all context changes; it could be de-growth with context changes; it could be strong sustainability with current preferences, a change in $\gamma$, $\varepsilon$, or $\zeta$, all preference parameters related to a sense of concern or responsibility with all levels of aversion, and all context changes.

With a focus on consumption and environment (here, Sen; Gini-equitable in consumption and environment use), a case consistent with the egalitarian approach could be weak sustainability with all preference parameters related to a sense of concern or responsibility and small aversion to inequality; could be a-growth with current preferences, a change in $\gamma, \delta$, or $\zeta$, all preference parameters related to a sense of concern or responsibility with small or medium aversions, and all 
context changes; could be de-growth with context changes; or could be strong sustainability with current preferences, a change in $\gamma, \delta, \varepsilon$, or $\zeta$, and all preference parameters related to a sense of concern or responsibility, with all levels of aversion, for all context changes.

A case consistent with the contractarian approach (here, Rawls; MaxMin-equitable in consumption, environment use, or welfare) could be a-growth with a change in $\gamma$, improved technology and modified consumption; could be de-growth with improved technology and modified consumption; or could be strong sustainability with a change in $\gamma$, improved technology and modified consumption. In summary, by considering the effects of modified consumption, decreased population and improved technology, the ordering of sustainability paradigms is strong sustainability $>$ a-growth $>$ de-growth $>$ weak sustainability. Note that de-growth is inconsistent with any equity approach, by disregarding these effects. Although modified consumption and improved technology are ranked better than a sense of responsibility for future generations (i.e., $\alpha=$ $\theta>\gamma>(\beta, \gamma, \delta)$ with all $(\varepsilon, \zeta)=\eta>\delta=\zeta=$ current preferences $>\varepsilon)$, the ordering of value changes is $\gamma$ $>\delta=\zeta>\varepsilon$.

Table 7. Consistency with equity approaches. $\mathrm{Ut}=$ utilitarian approach, $\mathrm{Dw}=\mathrm{Dworkin}$ egalitarian approach, $\mathrm{Ar}$ = Arneson egalitarian approach, $\mathrm{Se}=$ Sen egalitarian approach, $\mathrm{Co}=$ contractarian approach, All = all equity approaches, blank = inconsistent with any equity approach. Sustainability paradigms: WS, weak sustainability; AG, a-growth; DG, de-growth; SS, strong sustainability.

\begin{tabular}{|c|c|c|c|c|c|c|c|c|c|}
\hline & $\mathrm{CP}, \zeta$ & $\beta$ & $\gamma$ & $\delta$ & $\varepsilon$ & $\begin{array}{c}\beta, \gamma, \delta \text { with } \\
\text { all } \varepsilon, \zeta\end{array}$ & $\alpha, \theta$ & $\eta$ & Rank \\
\hline WS & & & & & & Dw-Ar-Se & & & IV \\
\hline AG & Dw-Ar-Se & & All & Ut-Dw-Se & & Dw-Ar-Se & Dw-Ar-Se-Co & Dw-Ar-Se & II \\
\hline DG & & & & & & & Dw-Ar-Se-Co & Dw-Ar-Se & III \\
\hline SS & Dw-Ar-Se & & All & Ut-Dw-Se & Dw-Ar-Se & Dw-Ar-Se & Dw-Ar-Se-Co & Dw-Ar-Se & I \\
\hline Rank & IV & & II & IV & V & II & I & III & \\
\hline
\end{tabular}

Expected insights (to be taken as a validation of the model):

1. An inter-generational sense of responsibility $(\gamma)$ is the most important feature, although a concern for non-OECD countries $(\delta)$ also leads to sustainability with a-growth and strong sustainability.

2. A population reduction makes de-growth feasible.

3. Improved technology makes a-growth more feasible, and to a greater extent than with a population reduction.

4. Modified consumption makes de-growth more feasible.

5. Strong sustainability reduces inequality in consumption, environment use, and welfare in all scenarios, apart from welfare in a case with a concern for non-OECD countries $(\delta)$.

Unexpected insights (to be taken as findings from the model):

1. Aversion to inequality for non-OECD countries $(\varepsilon)$ is more important than aversion to inequality for future generations $(\zeta)$, although people of non-OECD countries pay more with larger $\varepsilon$ than with larger $\zeta$, whereas the opposite holds for people in OECD countries.

2. An increase in aversion to inequality for either non-OECD countries $(\varepsilon)$ or for future generations $(\zeta)$ makes a-growth more feasible in cases with a sense of responsibility for nature $(\beta)$ and current $(\delta)$ and future $(\gamma)$ generations.

3. Modified consumption makes strong sustainability more feasible.

4. In a case with a sense of responsibility for both nature $(\beta)$ and current $(\delta)$ and future $(\gamma)$ generations, a-growth and strong sustainability lead to feasible sustainability conditions that provide similar welfare for OECD and non-OECD countries, despite differences in the representative individuals, if aversion to inequality both for non-OECD countries $(\varepsilon)$ and for future generations is $(\zeta)$ small.

5. A large sense of responsibility for nature $(\beta)$ provides no feasible solutions for all sustainability paradigms. 
Weaknesses of the present study:

- Environmental free-riding (i.e., some individuals pay for the consequences of the environmental use by other individuals) is not modelled (e.g., in Appendix II, $E_{\mathrm{N}}$ and $E_{\mathrm{S}}$ are replaced by $E_{\mathrm{C}}=\sum p_{\mathrm{i}} E_{\mathrm{i}}$ ). However, welfare in OECD countries depends on welfare in nonOECD countries, which in turn depends on their environmental use (i.e., $U_{\mathrm{N}}$ depends on $U_{\mathrm{S}}$, which depends on $E_{\mathrm{S}}=2 \eta-E_{\mathrm{N}}$ and $E_{\mathrm{S}}=\eta / p_{\mathrm{S}}-\left(p_{\mathrm{N}} / p_{\mathrm{S}}\right) E_{\mathrm{N}}$ for non-weighted and weighted sustainability conditions, respectively).

- The institutionalisation of sustainability (i.e., implementation of technical, legal, and moral systems; development of organisations, boards, and offices to implement sustainability strategies; Ott, 2014) is not discussed. However, most operational conditions that would lead towards sustainability are identified (e.g., $\delta$ should be preferred to $\gamma$ ).

- Feasibility was defined based on arbitrary thresholds (i.e., a welfare reduction larger than $25 \%$ was defined as unfeasible). However, comparisons between value changes and the four sustainability paradigms are independent of these thresholds (e.g., a-growth and strong sustainability should be preferred to weak sustainability, which should be preferred to degrowth).

Strengths of the present study:

- The framework combines sustainability paradigms and equity approaches to find a theoretically coherent solution that can lead to sustainability or an operationally implementable policy that will lead to sustainability.

- The results confirm insights in the literature on context changes such as modified consumption and improved technology (Zagonari, 2015), but they also expand these insights to include value changes by distinguishing among four sustainability paradigms and by considering proportions of the global population in OECD and non-OECD countries and the dynamics of these populations.

- Neither a top-down nor a bottom-up approach to preference changes is suggested; instead, the most important features linked to each sustainability paradigm and equity approach are identified.

Therefore, in normative terms, if the a priori commitment is a consistent sustainability paradigm, this study suggests a focus on $\gamma$ (if a-growth or strong sustainability is adopted), $\delta$ (if a-growth is adopted), $\varepsilon$ (if strong sustainability is adopted), $\zeta$ (if strong sustainability is adopted), all senses of concern or responsibility combined (if a-growth or strong sustainability is adopted), and all context changes (if a-growth, de-growth, or strong sustainability is adopted). Moreover, in normative terms, if the a priori commitment is a consistent equity approach, this paper suggests a focus on $\gamma$ (in the case of utilitarian, egalitarian, or contractarian approaches), $\delta$ (in the case of utilitarian or egalitarian approaches), $\varepsilon$ (in the case of an egalitarian approach), and $\zeta$ (in the case of an egalitarian approach). Finally, in positive terms, the most effective sustainability conditions, regardless of consistency of the adopted paradigm or approach, appear to be $\gamma>\delta>\varepsilon>\zeta$ for value changes and a-growth $>$ strong sustainability $>$ de-growth $>$ weak sustainability for sustainability paradigms.

\section{Conclusions}

Three main novel findings were obtained in this study. First, weak sustainability and de-growth, which include the constraint $U_{\mathrm{F}} \geq U_{\mathrm{C}}$, are both theoretically problematic to sustain and empirically difficult to implement, since the happiness or needs of future generations, which depend on spatial and temporal variations in culture, cannot be easily defined or measured. Second, sustainability conditions cannot be achieved by relying only on a sense of responsibility for nature, but instead must rely on a concern for both future generations and non-OECD countries. In other words, relying on a sense of responsibility for nature, which only indirectly affects future generations, is theoretically ineffective; indeed, welfare losses in the absence of concern for future generations and non-OECD countries are large under all paradigms, although sustainability conditions differ among the paradigms. Relying on a sense of responsibility for nature is also operationally difficult; for 
example, agreements between religions are difficult to achieve, since their philosophies have evolved from different cultural backgrounds and histories. Third, the perspective of a single individual (i.e., the focus is on individual sustainability) and a representative individual (i.e., the focus is on overall sustainability) that are assumed by a-growth and strong sustainability, respectively, leads to sustainability at similar levels of per capita consumption and use of the environment by both OECD and non-OECD countries.

The main theoretical good news from this study is that the ecological debt (i.e., the current average ecological footprint of 2.79 ha is larger than the long-run equilibrium value at $1.7 \mathrm{ha}$ ), and can be paid in full by the current generation if there is significant concern for future generations and nonOECD countries, although the welfare burden depends on the possible preference changes and the distribution of this burden depends on the adopted sustainability paradigms.

The main operational good news is threefold. First, sustainability can be achieved at current preferences with reasonable burdens for current generations in OECD and non-OECD countries if a-growth or strong sustainability is adopted. Second, improved technology and modified consumption could increase welfare for the current non-OECD generation compared with the status quo. Third, whenever a feasible solution exists, the sustainability burden on current OECD generations is proportionally larger than that on current non-OECD generations.

The main theoretical bad news is that two of the four sustainability paradigms are not internally consistent: weak sustainability was never Kaldor-Hicks efficient (with and without context changes), whereas de-growth was never MaxMin-equitable (without context changes).

The main operational bad news is threefold. First, achieving sustainability implies an increase in inter-generational inequality: the current unsustainable representative individual for the world is closer to the future sustainable representative individual than to the current sustainable representative individual in terms of both consumption and environment use in all sustainability paradigms. Second, reducing the world's population, as endorsed by the de-growth paradigm, is not enough to achieve sustainability, although the current non-OECD generation would not decrease its status quo welfare if strong sustainability or de-growth were adopted. Third, if the suggested changes in preferences related to a sense of concern or responsibility are interpreted as changes in the budget share, sustainability conditions appear difficult to achieve.

Three main developments from this paper seem to be particularly promising. First, it should be possible to move from aggregated data to more detailed data on consumption levels. This would allow the inclusion of expenditures for health and education, possibly at a national level. Second, it should be possible to enhance the current one-shot model to produce a dynamic model, which would allow an investigation of issues related to overlapping generations. Third, moving from aggregated data to more detailed data on the direct and indirect use of Earth's resources would allow the framework to account for both pollution production and resource use, possibly at a national level.

\section{Appendix I}

$\alpha_{\mathrm{F}}$ : the future preference for consumption

$\alpha_{N}$ : the preference for consumption in OECD countries

$\alpha_{S}$ : the preference for consumption in non-OECD countries

$\beta_{\mathrm{N}}$ : the degree of concern for nature in OECD countries

$\beta_{\mathrm{S}}$ : the degree of concern for nature in non-OECD countries

$\gamma_{\mathrm{N}}$ : the degree of concern for future generations in OECD countries

$\gamma_{S}$ : the degree of concern for future generations in non-OECD countries

$\delta_{N}$ : the degree of concern for the current non-OECD generation in OECD countries

$\varepsilon$ : the degree of aversion to intra-generational inequality

$\zeta$ : the degree of aversion to inter-generational inequality

$\eta$ : per capita equilibrium use of the environment consistent with the current world population

$\theta_{\mathrm{N}}$ : the use of the environment for each consumption unit for the OECD current generation 
$\theta_{\mathrm{s}}$ : the use of the environment for each consumption unit for the non-OECD current generation

$\theta_{\mathrm{F}}$ : the use of the environment for each consumption unit for the future generation

$E_{\mathrm{C}}$ : population-weighted per capita use of the environment by the current generation

$E_{\mathrm{F}}$ : per capita use of the environment by the future generation

$E_{\mathrm{N}}$ : per capita use of the environment in the current OECD generation

$E_{\mathrm{S}}$ : per capita use of the environment in the current non-OECD

$p_{\mathrm{N}}$ : proportion of the global population in the OECD countries

$p_{\mathrm{S}}$ : proportion of the global population in the non-OECD countries

$U$ : overall utility as dependent on consumption

$U_{\mathrm{C}}$ : population-weighted utility of the current generation as dependent on consumption

$U_{\mathrm{F}}$ utility of the future generation as dependent on consumption

$U_{\mathrm{N}}$ : utility of the current OECD generation as dependent on consumption

$U_{\mathrm{S}}$ : utility of the current non-OECD generation as dependent on consumption

$W$ : overall welfare as dependent on environment use

$W_{\mathrm{C}}$ : population-weighted welfare of the current generation as dependent on environment use

$X_{\mathrm{C}}$ : population-weighted per capita consumption in the current generation

$X_{\mathrm{F}}$ : per capita consumption in the future generation

$X_{\mathrm{N}}$ : per capita consumption in the OECD current generation

$X_{\mathrm{S}}$ : per capita consumption in the non-OECD current generation

\section{Appendix II}

In the case of $n$ countries at a similar development level, and which share a common environment (e.g., a closed sea), the model changes as follows:

$$
\begin{gathered}
U F=X F^{\alpha \mathrm{F}} \text { with } \alpha_{\mathrm{F}}=\frac{1}{n} \sum_{i}^{n} \alpha_{i} \text { and } X F=\eta / \theta_{\mathrm{F}} \text { with } \theta_{\mathrm{F}}=\frac{1}{n} \sum_{i}^{n} \theta_{i} \\
U C_{i}=X_{i}^{\alpha i}\left(\sum p_{i} E_{i}\right)^{-\beta i} U F^{\gamma i}\left(\sum p_{j} U C_{j}\right)^{\delta i} \\
U C=\left[\left(\sum p_{i} U C_{i}\right)^{1-\varepsilon}\right]^{1 /(1-\varepsilon)} \\
U=\left[U C^{1-\zeta}+U F^{1-\zeta}\right]^{1 /(1-\zeta)} \\
W C=\left[\left(\sum p_{i} E_{i}\right)^{1-\varepsilon}\right]^{1 /(1-\varepsilon)} \\
W=\left[E C^{1-\zeta}+E F^{1-\zeta}\right]^{1 /(1-\zeta)}
\end{gathered}
$$

Where $i$ refers to a sum which includes all $n$ countries, whereas $j$ refers to a sum which excludes country $i$. Note that this system of equations could be solved for $X_{i}$ to check for the existence of a sustainability solution at current preferences. Alternatively, it could be solved for a set of consumption preferences (i.e., $\alpha_{i}, \beta_{i}, \gamma_{i}, \delta_{i}, \varepsilon, \zeta$ ) at current consumption levels to check which country should change its preferences to a greater extent. Moreover, $U_{\mathrm{F}} \geq U_{i}$ could be used instead of $U_{\mathrm{F}} \geq U_{\mathrm{C}}$. Finally, the model could be solved in a cooperative context, in which $\delta_{i}$ could be positive. Alternatively, it could be solved in a non-cooperative context, in which $\delta_{i}$ is set to 0 .

\section{References}

Arneson, R.J. (1989) Equality and equal opportunity for welfare, Philosophical Studies 56: 77-93

Asheim, G.B., Mitra, T, Tungodden, B. (2012) Sustainable recursive social welfare functions, Economic Theory 49: 267-292

Aznar-Marquez, J., Ruiz-Tamarit, J.R. (2016) Environmental pollution, sustained growth, and sufficient conditions for sustainable development, Economic Modelling 54: 439-449

Caselles, A. (2013) An application of fuzzy cognitive maps to improve well-being, sustainability and the globalization process, Systems Research and Behavioural Science 30: 646-660 
Dworkin, R. (1981) What is equality? Part 2: Equality of resources, Philosophy and Public Affairs 10: $283-345$

Golub, A., Mahoney, M., Harlow, J. (2013) Sustainability and intergenerational equity: do past injustices matter? Sustainability Science 8: 269-277

Gosseries, A. (2008) On future generations' future rights, Journal of Political Philosophy 16: 446474

Habib, A. (2013) Sharing the Earth: sustainability and the currency of inter-generational environmental justice, Environmental Values 22: 751-764

Harsanyi, J. C. (1982) Morality and the theory of rational behaviour, in Sen, Amartya; Williams, Bernard, Utilitarianism and beyond, Cambridge: Cambridge University Press, pp. 39-62

Jain, P.; Jain, P. (2013) Sustainability assessment index: a strong sustainability approach to measure sustainable human development, International Journal of Sustainable Development and World Ecology 20: 116-122

Kallis, G. (2011) In defence of de-growth. Ecological Economics 70: 873-880

Kallis, G.; Kerschner, C.; Martinez-Alier, J. (2012) The economics of de-growth, Ecological Economics 84: 172-180

Kershnar, S., Purves, D. (2016) A new argument for the irrelevance of equality for intrinsic value, Philosophia (United States): 1-21

Kopnina, H. (2016) The victims of unsustainability: a challenge to sustainable development goals, International Journal of Sustainable Development and World Ecology 23: 113-121

Koukouzelis, K. (2012) Sustainable development, liberty, and global social justice, Public Reason 4: $165-181$

Lauwers, L. (2012) Intergenerational equity, efficiency, and constructability, Economic Theory 49: 227-242

Lomasky, L. (1987) Persons, Rights, and the Moral Community, New York: Oxford University Press

Nozick, R. (1974) Anarchy, State, and Utopia, New York: Basic Books

Ott, K. (2014) Institutionalising strong sustainability: a Rawlsian perspective, Sustainability (Switzerland) 6: 894-912

Pedersen, K.P. (2015) Religious ethics and the environment: a review essay, Journal of Religious Ethics 43: 558-585

Rawls, J. (1971) A Theory of Justice, Cambridge: Harvard University Press

Saniotis, A. (2012) Muslims and ecology: fostering Islamic environmental ethics, Contemporary Islam 6: 155-171

Schlör, H.; Fischer, W.; Hake, J.F. (2015) The system boundaries of sustainability, Journal of Cleaner Production 88: 52-60

Sen, A.K. (1993) Capability and well-being, in M. C. Nussbaum and A. K. Sen (eds), The Quality of Life, Oxford: Clarendon Press: 30-53

Van den Bergh, J.C.J.M. (2010) Externality or sustainability economics? Ecological Economics 69: 2047-2052

Van den Bergh, J.C.J.M. (2011) Environment versus growth - a criticism of "de-growth" and a plea for "a-growth", Ecological Economics 70: 881-890

Van der Werff, E., Steg, L., Keizer, K. (2013) It is a moral issue: the relationship between selfidentity, obligation-based intrinsic motivation and pro-environmental behaviour, Global Environmental Change 23: 1258-1265

Zagonari, F. (2015) Technology improvements and value changes for sustainable happiness: a cross-development analytical model, Sustainability Science 10: 687-698

Zagonari, F. (2016) Four sustainability paradigms for environmental management: a methodological analysis and an empirical study based on 30 Italian industries, Sustainability (Switzerland) 8: 504-537 


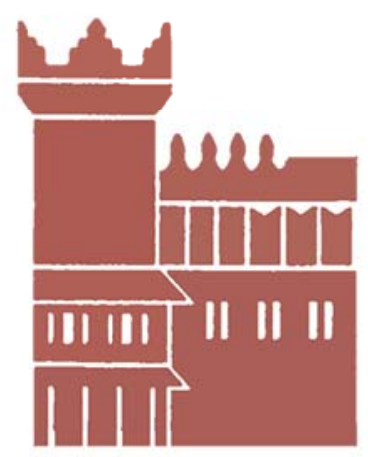

Alma Mater Studiorum - Università di Bologna DEPARTMENT OF ECONOMICS

Strada Maggiore 45

40125 Bologna - Italy

Tel. +39051 2092604

Fax +390512092664

http://www.dse.unibo.it 